\title{
The head and neck cancer immune landscape and its immunotherapeutic implications
}

\author{
Rajarsi Mandal, ${ }^{1,2,3}$ Yasin Şenbabaoğlu, ${ }^{4}$ Alexis Desrichard,,${ }^{1,3}$ Jonathan J. Havel, ${ }^{1,3}$ \\ Martin G. Dalin, ${ }^{1,3}$ Nadeem Riaz, ${ }^{3,5}$ Ken-Wing Lee, ${ }^{1,3}$ Ian Ganly, ${ }^{2}$ A. Ari Hakimi, ${ }^{6}$ \\ Timothy A. Chan, ${ }^{1,3,5}$ and Luc G.T. Morris , $^{1,2,3}$ \\ ${ }^{1}$ Human Oncology and Pathogenesis Program, ${ }^{2} \mathrm{Head}$ and Neck Service, Department of Surgery, \\ ${ }^{3}$ Immunogenomics and Precision Oncology Platform, ${ }^{4}$ Ludwig Collaborative/Swim Across America Laboratory, \\ Immunology Program and Department of Medicine, ${ }^{5}$ Department of Radiation Oncology, \\ ${ }^{6}$ Urology Service, Department of Surgery, Memorial Sloan Kettering Cancer Center, New York, New York, USA.
}

\begin{abstract}
Recent clinical trials have demonstrated a clear survival advantage in advanced head and neck squamous cell carcinoma (HNSCC) patients treated with immune checkpoint blockade. These emerging results reveal that HNSCC is one of the most promising frontiers for immunotherapy research. However, further progress in head and neck immuno-oncology will require a detailed understanding of the immune infiltrative landscape found in these tumors. We leveraged transcriptome data from $\mathbf{2 8 0}$ tumors profiled by The Cancer Genome Atlas (TCCA) to comprehensively characterize the immune landscape of HNSCC in order to develop a rationale for immunotherapeutic strategies in HNSCC and guide clinical investigation. We find that both $\mathrm{HPV}^{+}$and $\mathrm{HPV}^{-} \mathrm{HNSCC}$ tumors are among the most highly immune-infiltrated cancer types. Strikingly, HNSCC had the highest median Treg/CD8 ${ }^{+}$T cell ratio and the highest levels of CD56 dim NK cell infiltration, in our pan-cancer analysis of the most immune-infiltrated tumors. $\mathrm{CD8}^{+} \mathrm{T}$ cell infiltration and CD56 ${ }^{\mathrm{dim}} \mathrm{NK}$ cell infiltration each correlated with superior survival in HNSCC. Tumors harboring genetic smoking signatures had lower immune infiltration and were associated with poorer survival, suggesting these patients may benefit from immune agonist therapy. These findings illuminate the immune landscape of $\mathrm{HPV}^{+}$and $\mathrm{HPV}^{-} \mathrm{HNSCC}$. Additionally, this landscape provides a potentially novel rationale for investigation of agents targeting modulators of Tregs (e.g., CTLA-4, GITR, ICOS, IDO, and VEGFA) and NK cells (e.g., KIR, TIGIT, and 4-1BB) as adjuncts to antiPD-1 in the treatment of advanced HNSCC.
\end{abstract}

Conflict of interest: T.A. Chan is a cofounder of Gritstone Oncology and has ownership interest (including patents) in the same.

Submitted: August 1, 2016 Accepted: September 8, 2016 Published: October 20, 2016

Reference information: JCI Insight. 2016;1(17):e89829. doi:10.1172/ji.insight.89829.

\section{Introduction}

In recent years, immune checkpoint inhibitors have demonstrated promising response rates, and gained FDA approval for use, in patients with metastatic melanoma, non-small cell lung carcinoma (NSCLC), and renal cell carcinoma (1-5). These early successes have generated strong interest in the investigation of these therapies across multiple human cancers.

Head and neck squamous cell carcinoma (HNSCC) is the sixth most common cancer globally with 600,000 cases diagnosed annually, and mortality rates as high as $40 \%-50 \%(6-9)$. Traditional treatment for HNSCC is associated with substantial morbidity and toxicity. Recurrent and metastatic disease is usually incurable. These factors underscore the dire need for more effective therapies for patients with advanced HNSCC. No new targeted therapies have been approved for HNSCC for decades, other than cetuximab in 2006. Cetuximab affords only modest response rates $(10 \%-15 \%)$ as monotherapy, and there are no known biomarkers of response $(10,11)$. The complex mutational landscape of HNSCC explains, in part, the limited response rates of targeted therapies, as most tumors have multiple genetic drivers of oncogenesis and undergo evolution under the selective pressures of therapy (12-15).

Immunotherapy is a strategy that may potentially circumvent the need to target complex, redundant, and evolving molecular pathways. Preliminary data from trials of immune checkpoint inhibitors in the treatment of advanced HNSCC are promising (16-18). Therefore, at a minimum, there is a subset of 
HNSCCs that benefit from immunotherapeutic strategies. However, most HNSCCs are resistant to immunotherapy $a b$ initio. While the precise mechanistic basis remains unknown, this may be attributable to factors in the tumor microenvironment, such as a lack of appropriate rejection antigens, deficient immune surveillance, or the presence of immunosuppressive mediators.

The aim of this study was to analyze the transcriptomes of HNSCC tumors, integrated with mutation and copy number data, to illuminate the immune infiltrative landscape of HNSCC, the interplay between immune infiltration and molecular features such as HPV status, genetic alterations, neoantigen burden, mutational signatures of tobacco smoking, and the impact of these factors on clinical outcome. A more granular understanding of the immune infiltrative landscape in HNSCC may aid in developing the rationale for, and thereby guiding, ongoing clinical investigation within immuno-oncology.

\section{Results}

The immune microenvironment in HNSCC. We analyzed the RNA sequencing data from 280 HNSCCs profiled by TCGA (12). To deconvolute the levels of tumor-infiltrating immune cell populations and other immune signaling molecules, we used a previously described technique (19) employing single-sample gene set enrichment analysis (ssGSEA) (Figure 1A and Supplemental Table 1; supplemental material available online with this article; doi:10.1172/jci.insight.89829DS1). Based on immune cell populations, we subclassified HNSCCs into those with high or low overall immune infiltration (immune-high and immune-low). Phenotypically, tumor-associated lymphocytes may be either functionally active or inactive (secondary to exhaustion or anergy). To determine if differential immune cell populations correlated with levels of immune activation, we examined levels of the end-products or effectors of immunity, such as molecules released by activated $\mathrm{T}$ cells (granzymes and perforin), the cytolytic score (a surrogate measure of cytotoxic T lymphocyte activity developed by Rooney et al. in ref. 20), and enrichment of the IFN- $\gamma$ signaling pathway. We found that immune-high HNSCC tumors had universally elevated levels of immune activation, including granzyme A $\left(P=10^{-13}\right)$, granzyme $\mathrm{B}\left(P=10^{-12}\right)$, perforin $\left(P=10^{-13}\right)$, cytolytic score $(P=4$ $\left.\times 10^{-12}\right)$, and enrichment of the IFN- $\gamma$ signaling pathway $\left(P=7 \times 10^{-4}\right)$ (Figure 1B). Enrichment in tumor IFN- $\gamma$ signaling correlated strongly with levels of T cell infiltration $\left(r=0.74, P<10^{-4}\right)$ and overall immune cell infiltration $\left(r=0.76, P<10^{-4}\right)$ (Figure 1C). These data indicate that RNA sequencing-derived levels of immune cell infiltration are closely tied with levels of immune activity in the tumor microenvironment, and demonstrate a wide range in immune infiltration across head and neck tumors.

Interestingly, the $\mathrm{CD} 8^{+} /$Treg cell ratio was much higher in the immune-low population compared with the immune-high population $\left(P<1 \times 10^{-4}\right)$ (Figure 1B). This suggests that, despite the higher overall level of immune infiltration in the immune-high population, immune-high head and neck tumors appear to often be subjected to a higher degree of immunoregulatory influence. When stratifying HNSCC tumors by subsite, we observe a parallel trend. Tumors arising in the oropharynx display generally higher levels of $\mathrm{T}$ cell infiltration and immune activation, but also higher levels of immunoregulatory influence as represented by their comparatively lower $\mathrm{CD}^{+} /$Treg ratio (Figure 1D). These findings are consistent with the rich lymphoid tissue in the oropharynx, and may also reflect the high prevalence of HPV-associated cancers in this site (see HPV status below).

HNSCC can be subclassified into distinct molecular subtypes based on their expression profiles: atypical, basal, classical, and mesenchymal $(12,21,22)$. The atypical subtype contains the majority of HPV-positive tumors. The classical subtype is more associated with tobacco carcinogenesis, and closely resembles the classical subtype of lung squamous cell carcinoma $(12,22)$. We found that the atypical and mesenchymal subtypes had the highest degrees of immune infiltration and cytolytic activity, and classical, the lowest (Figure 1E).

Molecular correlates of immune infiltration. We then examined whether immune infiltration was associated with smoking history. There were marginal associations between clinical (self-reported) tobacco history and levels of T cell infiltration $(P=0.066)$ and overall immune infiltration $(P=0.034)$ (Supplemental Figure 1$)$. However, clinical smoking history may be less accurate than a molecular smoking signature. We therefore examined the association between molecular smoking signatures and immune correlates in HNSCC. There were strong inverse correlations between molecular tobacco signatures and levels of immune infiltration. Using the mutational signature framework defined by Alexandrov (23), we found that the proportion of mutational processes in each tumor attributable to tobacco smoking correlated inversely with the degree of T cell infiltrate $\left(r=-0.28, P=6 \times 10^{-6}\right)$, immune cell infiltrate $\left(r=-0.30, P=1 \times 10^{-6}\right)$, and IFN- $\gamma$ signaling 
A

Immune Subpopulation Single Sample Gene Set Enrichment
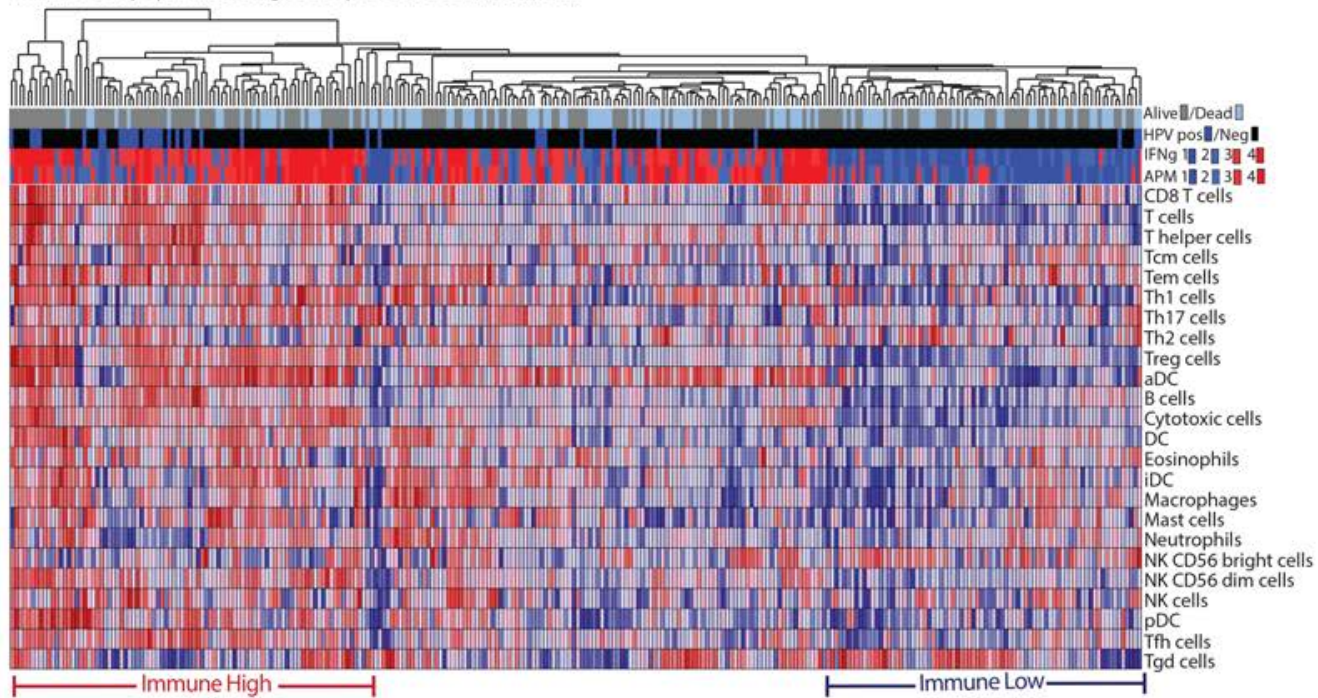

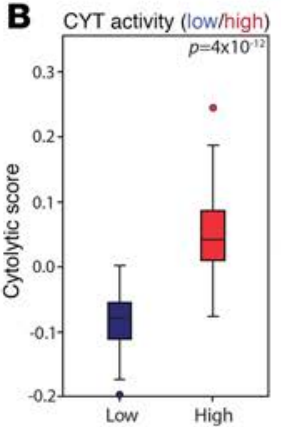

Immune infiltration
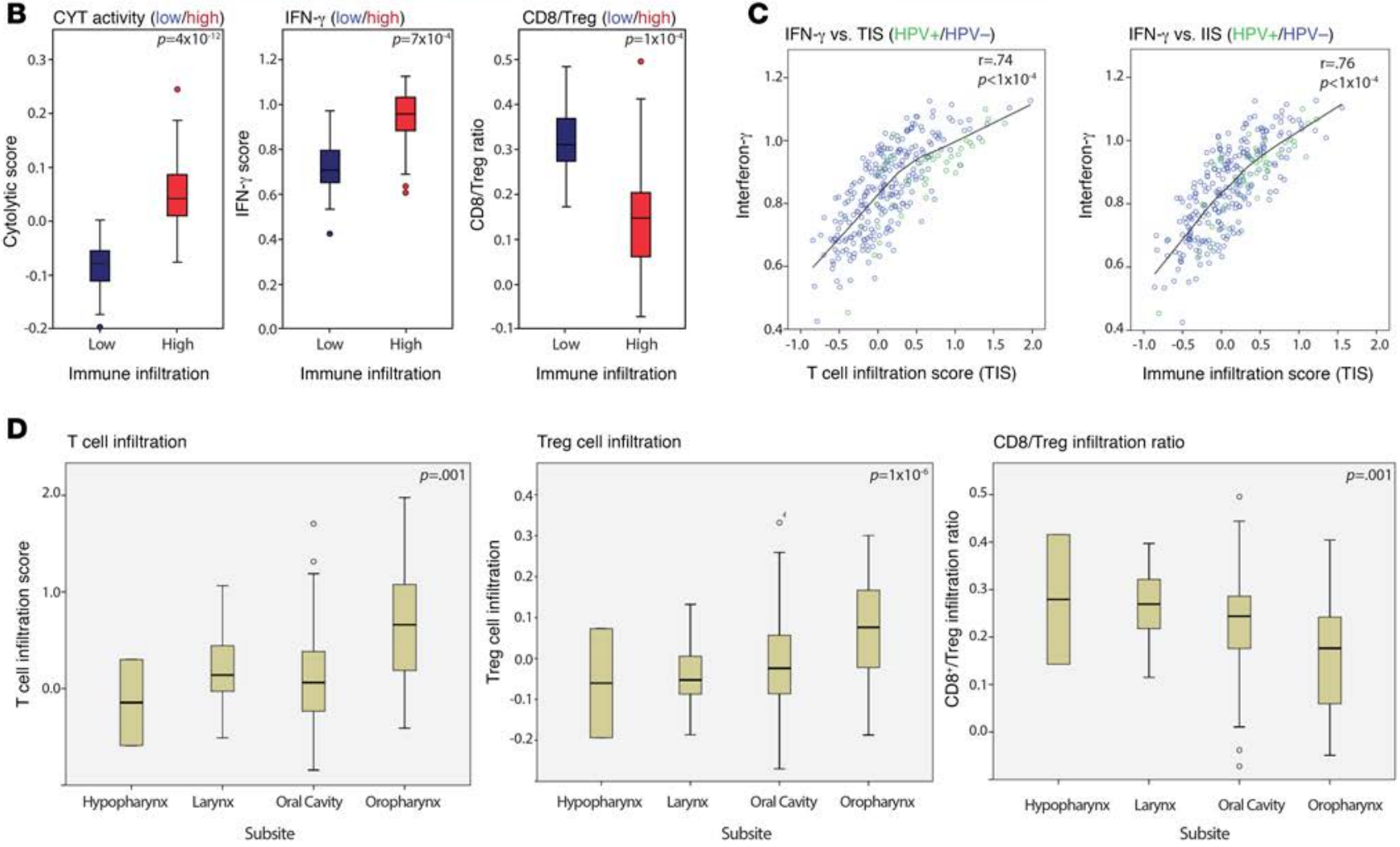

E
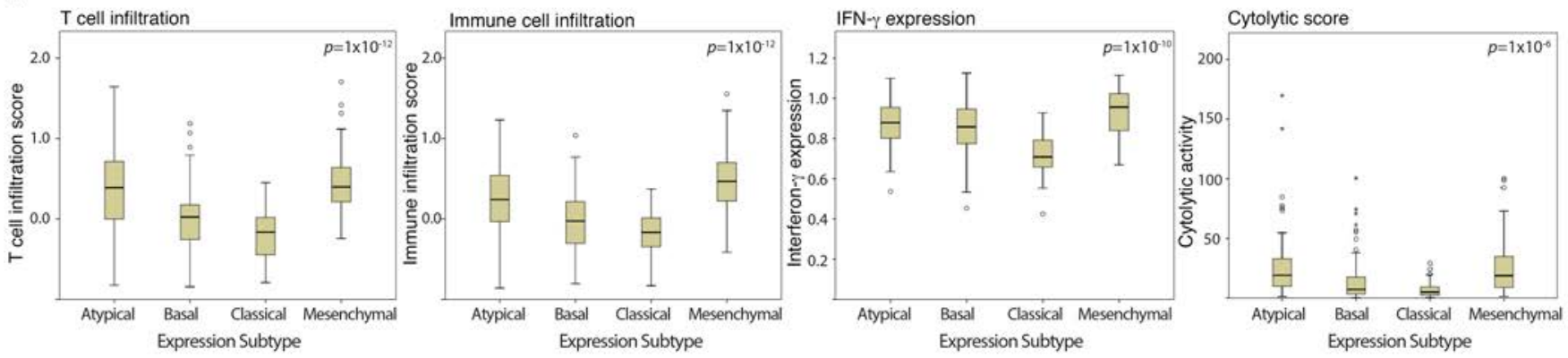
Figure 1. The immune landscape of head and neck squamous cell carcinoma (HNSCC). (A) Single-sample gene set enrichment analysis for 280 HNSCC tumor samples identifying relative expression of immune cell populations clustered into immune-high and -low phenotypes. (B) Relative cytolytic (CYT) activity, IFN- $\gamma$ expression, and $\mathrm{CD}^{+} /$Treg ratios between immune-high and -low tumors clustered by overall immune cell infiltration. (C) Correlations between relative IFN- $\gamma$ expression and immune infiltration characterized by T cell infiltration score (TIS) and immune cell infiltration score (IIS) in HPV-positive and -negative tumors. (D) Relative TIS, Treg, and CD8 ${ }^{+} /$Treg ratios stratified by head and neck tumor subsite. (E) Relative TIS, IIS, IFN- $\gamma$ expression, and CYT activity between HNSCC gene expression subtypes. All box-and-whisker plots represent values within the interquartile range (IQQR) (boxes) and $1.5 \times \mathrm{IQR}$ (whiskers). Outliers are plotted as values $>1.5 \times I Q R$ (circles) and $>3 \times I Q R$ (stars). All $P$ values for significance $(<0.05)$ represent comparisons via 2-tailed $t$ tests and 1-way ANOVA for continuous comparisons. All $r$ values represent Pearson correlation coefficients. $n=280$. APM, antigen-processing machinery.

$\left(r=-0.38, P=2 \times 10^{-10}\right)$. These correlations remained significant after adjusting for tumor HPV status (all $P$ $<10^{-4}$ ) in multivariable regression (Figure 2A and Supplemental Table 2). Alternatively, tumor exomes can be examined for levels of transversion mutations, consistent with tobacco carcinogenesis. We found that exomes marked by high levels of transversions had significantly lower levels of $\mathrm{T}$ cell infiltrate $(P=0.001)$, immune cell infiltrate $(P=0.001)$, and IFN- $\gamma$ signaling $\left(P=4 \times 10^{-7}\right)($ Supplemental Figure 2$)$. These data are consistent with prior evidence that tobacco smoking is associated with local immunosuppression and lower levels of cytotoxicity within the immune microenvironment (24). The exact mechanisms of smokingrelated immunosuppression are not well defined, but likely involve suppressive effects on NK cells, CD8 ${ }^{+}$ T cells, and DCs (24).

The tobacco mutational signature was positively correlated with tumor mutational load $(r=0.34, P=$ $\left.1.2 \times 10^{-8}\right)$. This association remained significant when adjusting for HPV status $\left(P=1.4 \times 10^{-8}\right)$ (Figure 2B). These data indicate that higher levels of tobacco-associated mutagenesis are associated with higher tumor mutational burden, consistent with results reported in lung cancer (25).

HPV status. HPV-positive and HPV-negative HNSCCs are genetically and molecularly distinct cancers $(12,13)$. We found that HPV-positive tumors were much more strongly immune infiltrated compared with HPV-negative tumors, with higher levels of T cell infiltration $\left(P=2 \times 10^{-9}\right.$; $\mathrm{CD}^{+} \mathrm{T}$ cells, $\left.P=1.7 \times 10^{-5}\right)$ and overall immune cell infiltration $\left(P=3 \times 10^{-4}\right)$ (Figure 3A). Consistent with higher infiltration by CD8 CTLs, HPV-positive tumors harbored higher levels of immune activation: specifically, perforin $(P=0.001)$, granzyme A $\left(P=3.6 \times 10^{-4}\right)$, and granzyme B $\left(P=1.9 \times 10^{-4}\right)$ expression (Figure $\left.3 \mathrm{~B}\right)$. The cumulative effect of these molecules as quantified by the cytolytic score $(20)$ was significantly higher $\left(P=10^{-6}\right)$ in HPVpositive tumors. These data illustrate that HPV-positive tumors have a distinct immune phenotype, marked with more immune infiltration and higher levels of $\mathrm{CD}^{+} \mathrm{T}$ cell activation.

At the same time, we observed significant immunoregulatory influences present in HPV-positive HNSCC. While PD-1 and PD-L1 expression was comparable in HPV-positive and HPV-negative tumors, there was significantly higher expression of the immunoinhibitory receptor CTLA-4 in HPV-positive tumors $(P=0.003)$ (Figure 3C). We observed a high positive correlation $(r=0.866, P<0.00001)$ between CTLA-4 expression and Treg infiltration, as expected, as these cells are known to highly express CTLA-4 (Supplemental Figure 3). In both HPV-positive and -negative tumors, we observed, positive correlations between PD-L1 expression and immune infiltration and activation metrics (Supplemental Figure 4). We also compared PD-1 and CTLA-4 expression between paired tumor and normal tissue samples available through the TCGA data set and found that expression of these receptors is, as expected, elevated in tumor tissue as compared with adjacent normal tissue (Supplemental Figure 5).

Similarly, HPV-positive tumors also contained higher levels of infiltrating Tregs, and higher Treg/ $\mathrm{CD}^{+} \mathrm{T}$ cell ratios. In fact, these levels of infiltration were the highest of 10 cancer types in our pancancer analysis (see Pan-cancer analysis below). Taken together, these data demonstrate that HPV-positive HNSCC tumors are characterized by elevated levels of immune infiltration in concert with high levels of immunoregulatory influences. These findings suggest that the addition of therapies targeting immunoregulatory elements of the HPV-positive tumor microenvironment may be particularly helpful in overcoming resistance to immune checkpoint blockade.

Pan-cancer immune landscape analysis. We analyzed immune cell infiltration and levels of immune activation in 10 cancer types with the highest overall immune infiltration scores (19). HPV-positive HNSCC displayed levels of immune infiltration that were among the highest of all cancer types, lower only than lung adenocarcinoma and kidney clear cell carcinoma. HPV-negative HNSCC also displayed high levels of immune infiltration, but lower than those of thyroid cancer, breast cancer, and melanoma (Figure 4A). Similar relative levels of immune infiltration were identified when examining $\mathrm{T}$ cell infiltration levels (Fig ure 4B). Cancer types with higher levels of T cell infiltration generally had higher levels of IFN- $\gamma$ signaling 
A

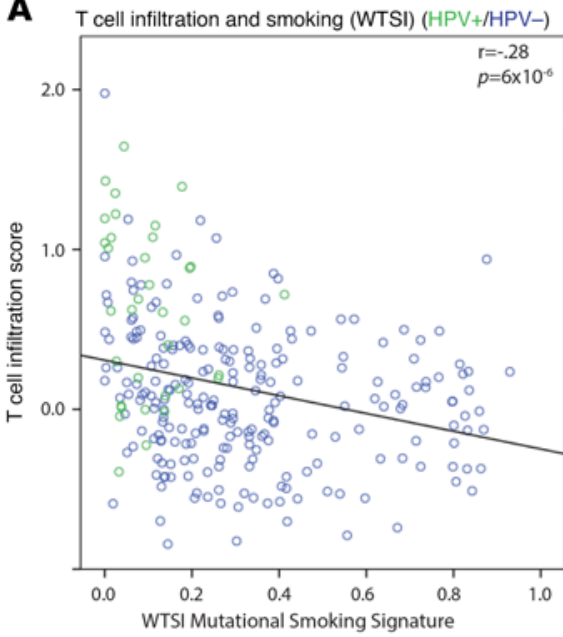

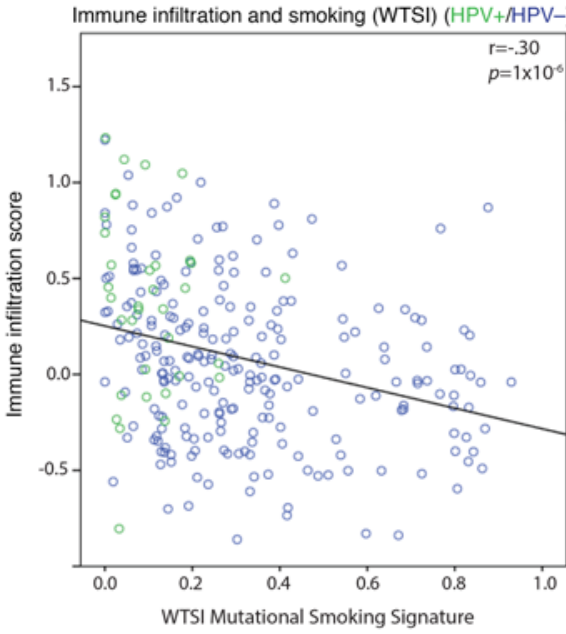

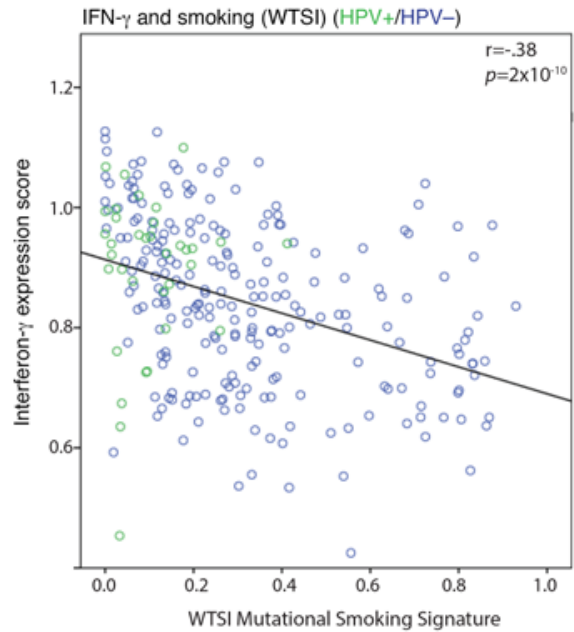

B Mutational burden and smoking (WTSI) (HPV+/HPV-)

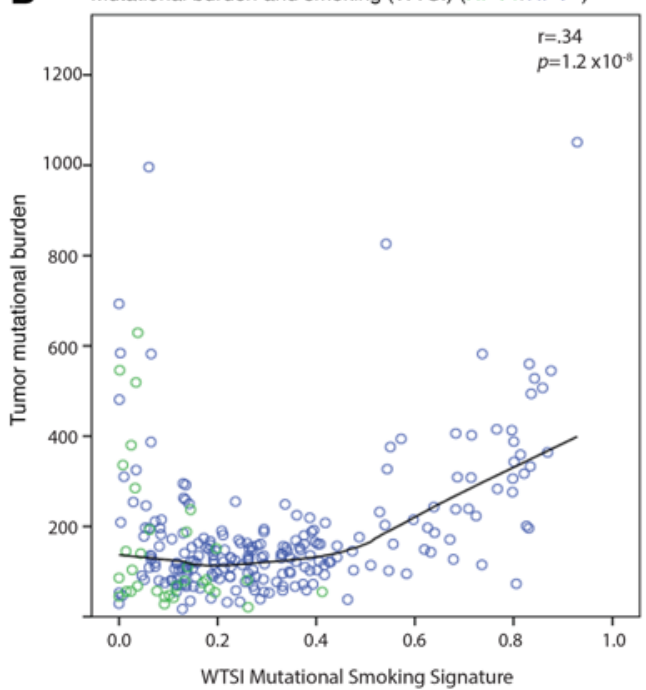

Figure 2. Immune infiltration and molecular smoking signatures. (A) T cell infiltration score (TIS), immune cell infiltration score (IIS), and IFN- $\gamma$ expression scores correlated with the Wellcome Trust Sanger Institute (WTSI) Mutational Smoking Signature Framework in HPV-positive (green) and -negative (blue) tumors. (B) Correlation between nonsynonymous tumor mutational burden and smoking signature quantified by the WTSI Mutational Smoking Signature Framework in HPV-positive (green) and -negative (blue) tumors. All $r$ values represent Pearson correlation coefficients. Two-tailed $P$ values are presented for significance $(<0.05) \cdot n=280$.

( $r=0.60, P=0.026$ ), with HPV-positive tumors again exhibiting among the highest levels of immune infiltration and adaptive immune response (Figure 4B).

Responses to immune checkpoint inhibition have been found to correlate with mutation count in several cancer types (25-27). This has been attributed to the creation of immunogenic peptides resulting from somatic mutations (neoantigens). These result when mutations lead to the generation of immunogenic neoepitopes presented to the immune system via MHC class I molecules. While there was no clear correlation across cancer types between levels of immune infiltration and nonsynonymous mutational burden (the total number of nonsilent missense mutations per tumor), both HPV-positive and HPV-negative tumors exhibited moderate-to-high mutation counts, comparable with those of other tumor types that have been found to respond to immune checkpoint blockade: melanoma, lung squamous cell carcinoma, and lung adenocarcinoma. The generally high levels of immune infiltration and mutational burden in HPV-positive and HPV-negative HNSCC together provide a strong rationale for investigation of immunotherapy in these tumors (17, 28-30) (Supplemental Figure 6).

Treg infiltration. Of the 10 cancer types, HPV-positive HNSCC had the highest levels of Treg infiltration, with HPV-negative HNSCC having the second highest (Figure 4C). This trend was recapitulated when examining the ratio of Treg/CD8 ${ }^{+} \mathrm{T}$ cells, which depicts the relative balance of extrinsic immunoregulatory influence in the tumor microenvironment (Figure 4D). These data suggest that HNSCC, especially HPV-positive tumors, are marked with both high levels of immune cell infiltration and high degrees of immunosuppression. This provides a strong rationale for the treatment of these tumors with 


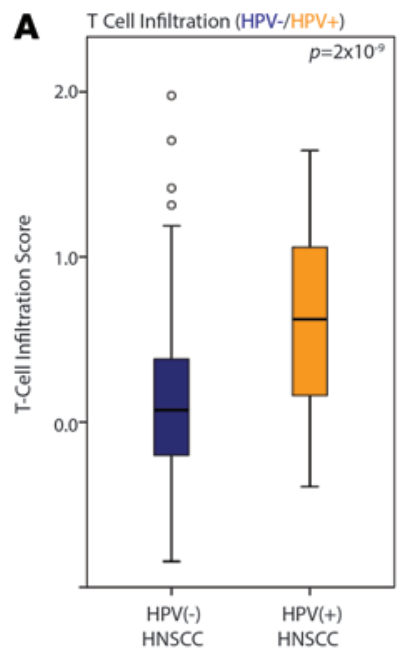

B Granzyme A Expression (HPV-/ $/ \mathrm{HPV+}$

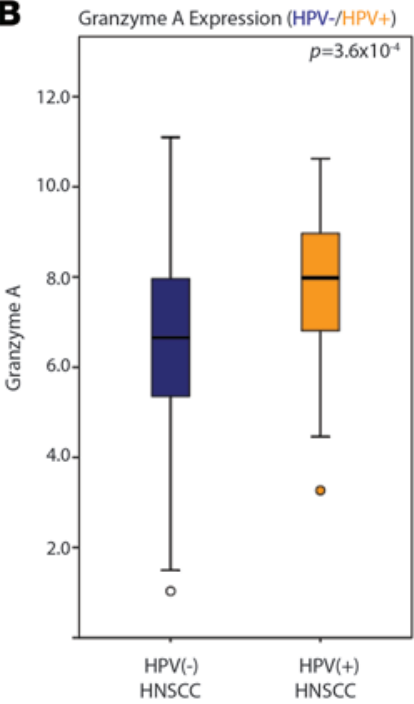

C

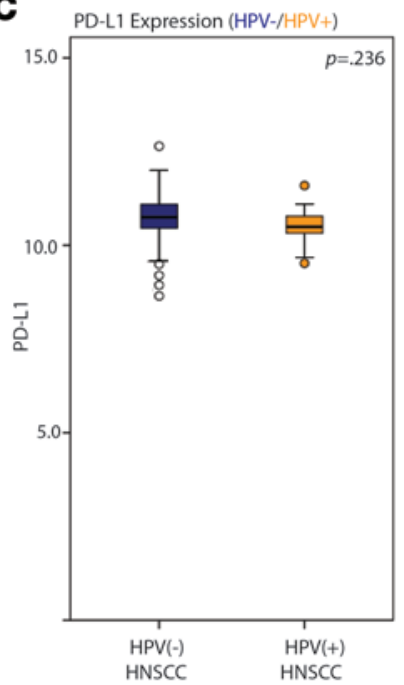

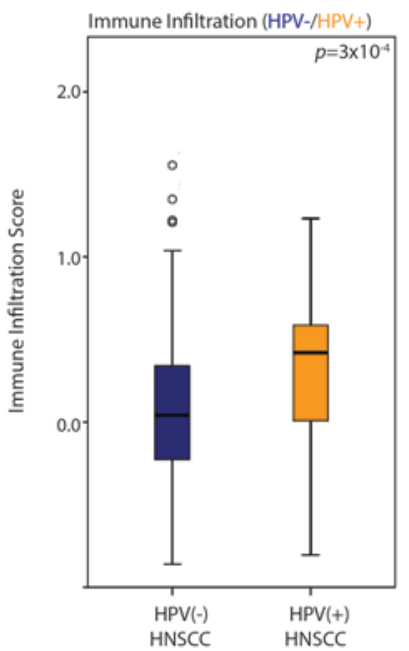
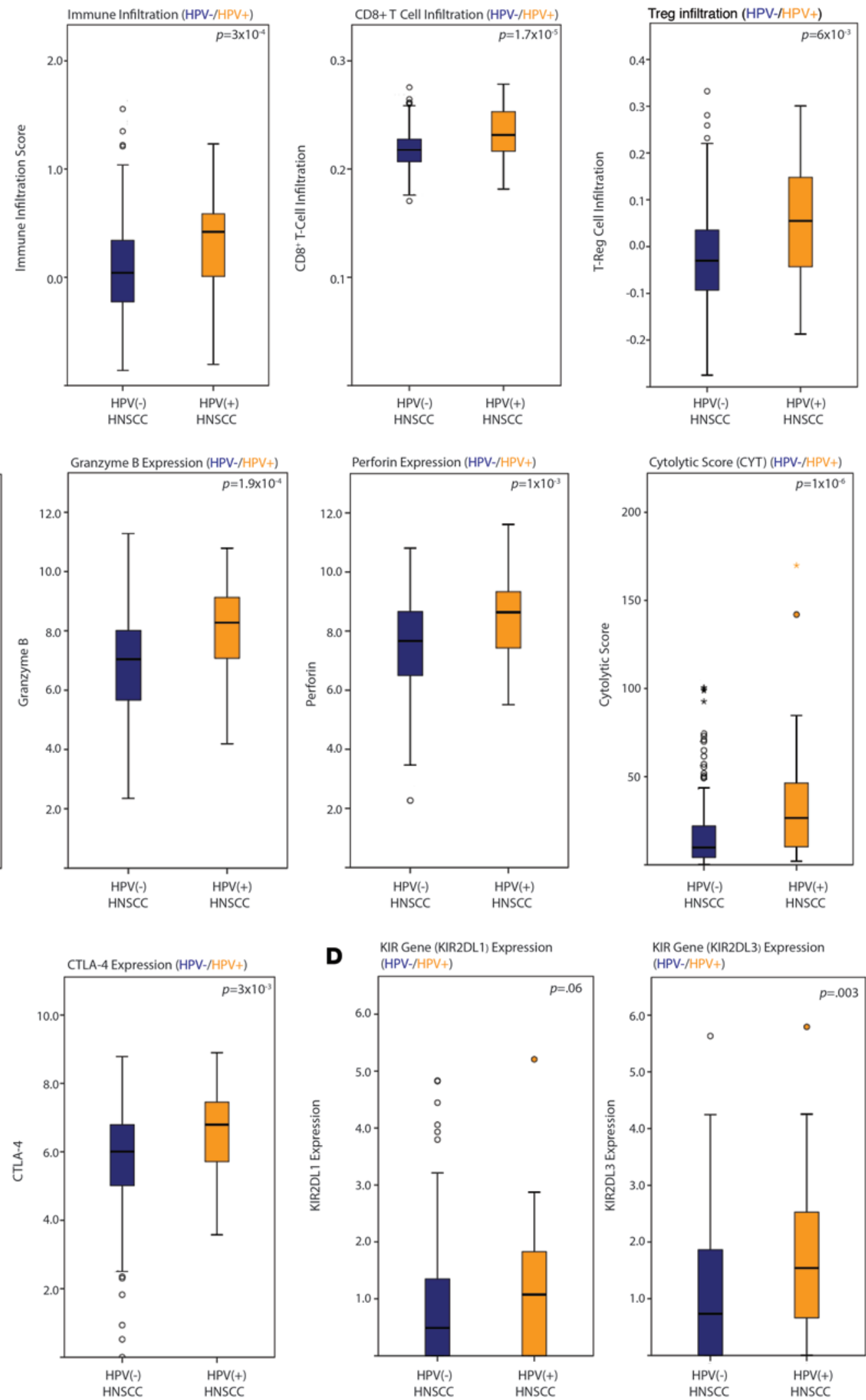

Figure 3. Immune infiltration in HPV-positive and -negative head and neck squamous cell carcinoma (HNSCC). (A) Relative T cell infiltration score (TIS), immune cell infiltration score (IIS), CD8 ${ }^{+}$T cell infiltration, and Treg infiltration between HPV-negative and -positive squamous cell tumors. (B) Relative expression of immune cell-derived cytotoxic effectors granzyme A, granzyme B, perforin, and their composite effect quantified by their cytolytic (CYT) score between HPV-negative and -positive squamous cell tumors. (C) Relative expression of PD-L1 and CTLA-4 between HPV-negative and -positive squamous cell tumors. (D) Relative median expression of killer cell immunoglobulin-like receptor (KIR) inhibitory genes, KIR2DL1 and KIR2DL3, in HPV-positive and -negative tumors. All box-and-whisker plots represent values within the interquartile range (IQR) (boxes) and $1.5 \times I Q R$ (whiskers). All $P$ values for significance $(<0.05)$ represent comparisons via 2-tailed $t$ tests. $n=280$. 
immunotherapy modalities, specifically targeting Tregs, with immune checkpoint blockade and other means (discussed further below).

NK cell subpopulations. The notion that NK cells function solely as effectors of innate immunity has been challenged in recent years. Increasing evidence supports their role in influencing the key components of adaptive immunity. This is mediated largely by their potent and early secretion of IFN- $\gamma$, which helps shape the immune tumor microenvironment by activating the effectors of adaptive immunity, particularly Th1 and myeloid cells $(31,32)$. This ability to influence adaptive immunity as well as their innate tumor cytolytic capability implicates NK cells as key effectors of antitumor immunity. As a result, the blockade of immunoregulatory NK cell checkpoint receptors has garnered significant interest (32-34). Agents (e.g., lirilumab) targeting NK cell inhibitory receptors, such as killer cell immunoglobulin-like receptor (KIR), are the subject of intense preclinical/clinical investigation as a monotherapy or in combination with other checkpoint inhibitors.

We analyzed levels of NK cell infiltration in HNSCC. The CD56 bright subpopulation is predominantly found in lymph nodes and peripheral blood and believed to be the likely precursor to CD56 ${ }^{\mathrm{dim}}$ cells, which are far more cytotoxic and play a critical role in antitumor immunity (35). Strikingly, HPV-positive and HPV-negative HNSCC tumors had the numerically highest levels of infiltration with CD56 ${ }^{\text {dim }}$ NK cells, compared with other highly immune-infiltrated cancer types (Figure 4E).

CD56 ${ }^{\text {dim }}$ NK cells are inhibited by KIRs, which are absent on CD56 ${ }^{\text {bight }}$ NK cells. CD56 $6^{\text {dim }}$ NK cell infiltration correlated strongly with overexpression of the KIR inhibitory receptor genes KIR2DL1 ( $r=$ $\left.0.36, P=4 \times 10^{-10}\right)$ and KIR2DL3 $\left(r=0.49, P=1 \times 10^{-13}\right)$. KIR gene expression was higher in HPV-positive tumors (KIR2DL1, $P=0.06$; KIR2DL3, $P=0.003$ ) (Figure 3D). These data suggest that HNSCC tumors are prime candidates for trials of anti-KIR antibodies.

Taken together, these data reveal that HNSCC tumors, both HPV-positive and HPV-negative, are infiltrated with Tregs and KIR-overexpressing CD56 ${ }^{\mathrm{dim}} \mathrm{NK}$ cells, to a higher degree than other cancer types. These findings suggest that head and neck tumors possess an immune landscape that is poised to respond to immunotherapeutic approaches that block inhibitory signals to T cells and NK cells, such as immune checkpoint inhibitors, including emerging antibodies blocking KIR signaling as well as other NK cell receptor targets.

Prognostic implications of immune infiltration in HNSCC. The prognosis of HNSCC varies significantly based on HPV status: patients with HPV-positive tumors experience higher rates of cure and superior overall survival (OS) (36). There are many molecular factors believed to contribute to improved survival in HPV-positive HNSCC, many of which are attributable to differences in genetic alterations. It is possible that major differences in immune infiltration may also mediate part of these survival differences. We therefore analyzed the prognostic impact of immune-related diversity in HNSCC, controlling for HPV status with multivariable regression. Measures of overall immune infiltration have been strongly associated with survival in other types of cancer (37), but have not yet been studied in detail in HNSCC.

Patients with highly immune-infiltrated tumors had significantly superior OS. Tumors with high levels of immune cell (hazard ratio $[\mathrm{HR}]=0.66,95 \% \mathrm{CI} 0.46-0.94, P=0.023$ ), $\mathrm{T}$ cell $(\mathrm{HR}=0.53,95 \% \mathrm{CI}$ $\left.0.37-0.76, P=6 \times 10^{-4}\right)$, and $\mathrm{CD}^{+} \mathrm{T}$ cell $(\mathrm{HR}=0.67,95 \% \mathrm{CI} 0.47-0.96, P=0.029)$ infiltration were associated with better OS. When controlling for HPV status in multivariable Cox regression, the associations remained significant for T cell $(P=0.013)$ and $\mathrm{CD}^{+} \mathrm{T}$ cell $(P=0.03)$ infiltration, and were borderline significant for overall immune cell infiltration $(P=0.10)$ (Figure 5A and Supplemental Table 3), indicating that these immune populations have independent prognostic significance, and do not simply reflect the results of HPV-positive tumors being more highly immune-infiltrated.

We performed additional modeling to examine whether the prognostic impact of immune infiltration and HPV status modulate one another, using an interaction term in multivariable regression. There was a significant interaction between immune infiltrate and HPV status (odds ratio $[\mathrm{OR}]=1.7, P=0.044$ ) (Supplemental Table 4). This indicates that HPV status modulates the prognostic effects of immune infiltration: levels of immune infiltration affect prognosis significantly more strongly in HPV-negative tumors than in HPV-positive tumors.

In general, infiltration with immune subpopulations involved in the host adaptive immune response tended to correlate with improved survival, whereas subpopulations involved in the innate immune response were negatively correlated with survival (Figure $5 \mathrm{~B}$ ). Indeed, the strongest negative prognostic factor in HNSCC was infiltration with Th2 cells $(P=0.006)$ (Figure $5 \mathrm{~B})$. While adaptive immunity has been 
A Ten most immune-infiltrated tumors

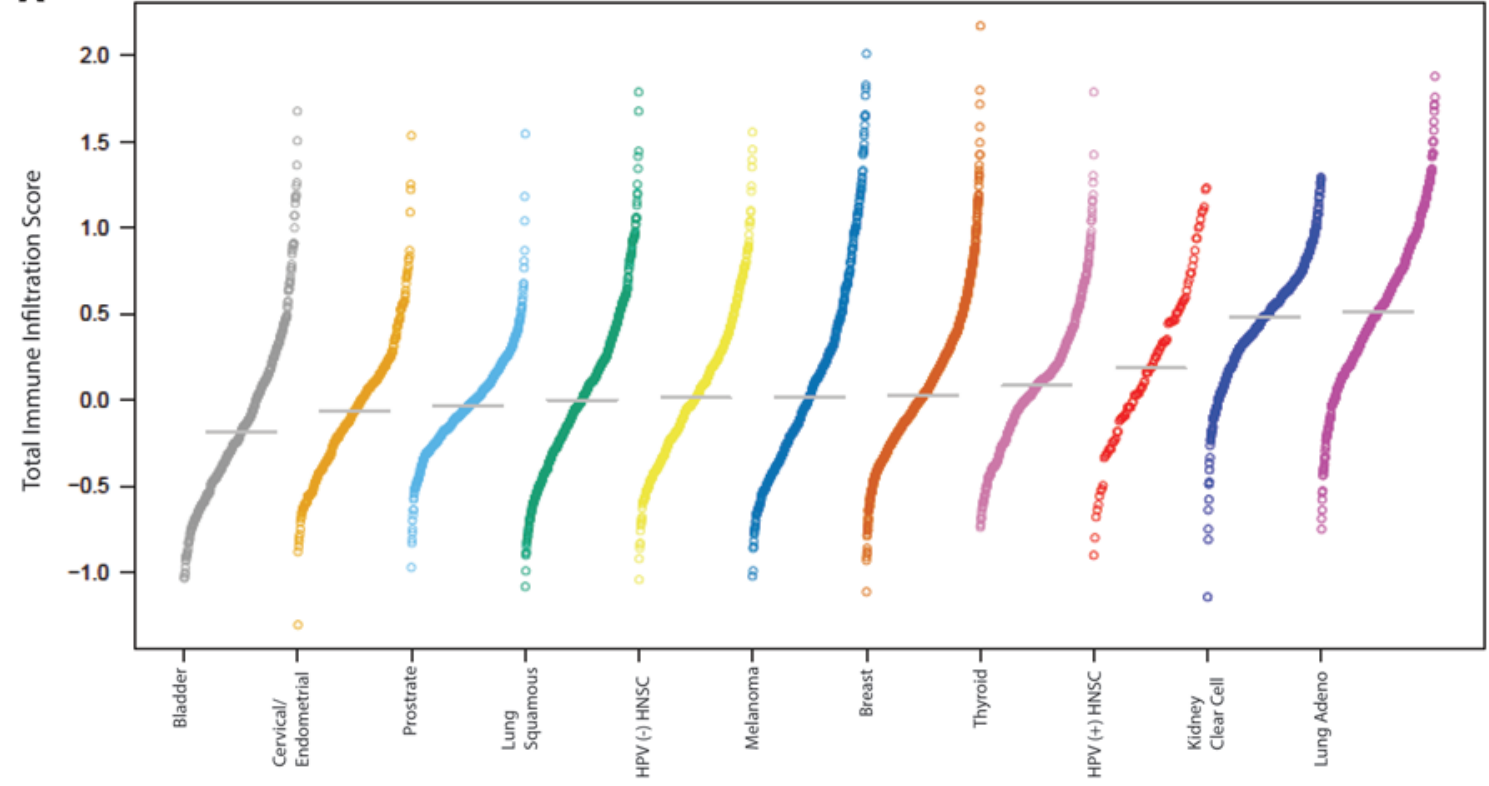

B

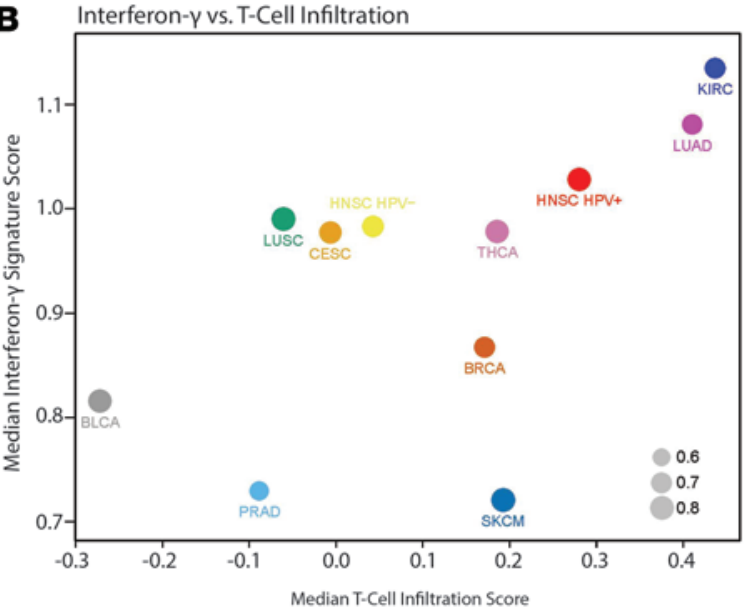

C T-Regulatory Cell Infiltration

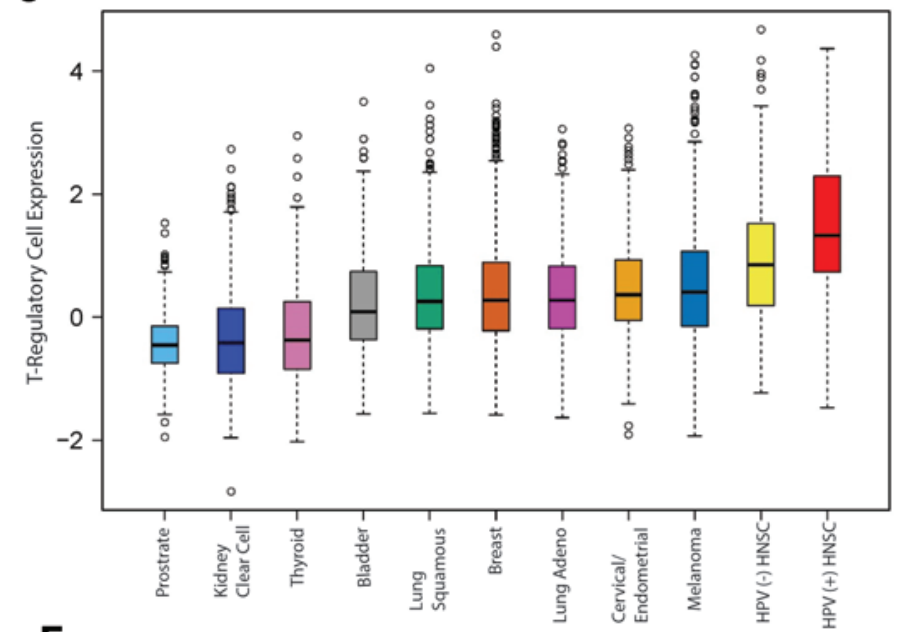

E

E $\quad \operatorname{CD}^{\mathrm{dim}} \mathrm{NK}$ Cell Infiltration

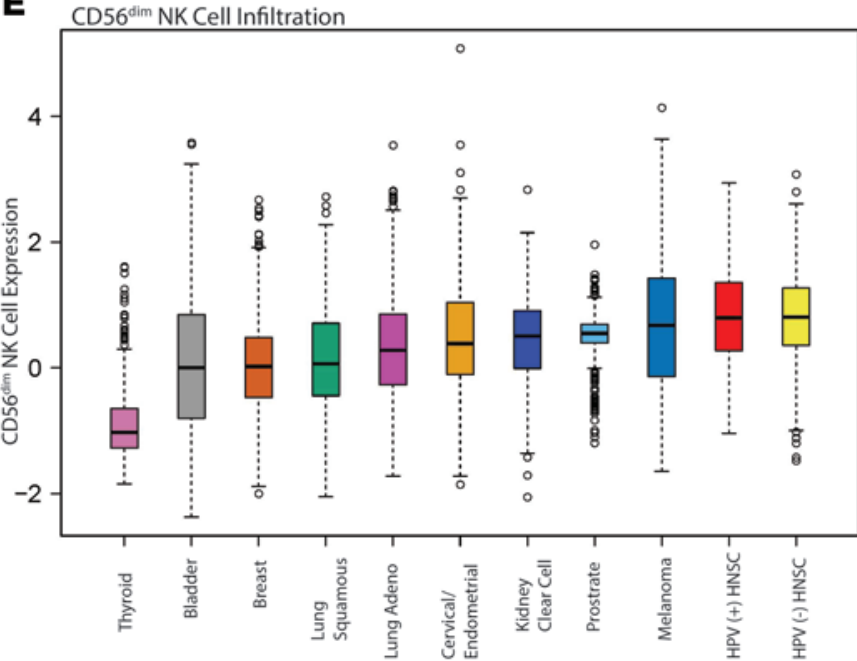

Figure 4. Pan-cancer immune infiltration analysis. (A) Relative median immune infiltration across the 10 most immune-infiltrated tumors in increasing order including HPV-positive and -negative HNSCC tumors. (B) Correlations between IFN- $\gamma$ expression scores and median T cell infiltration score (TIS) across the 10 most immuneinfiltrated cancer types including HPV-positive and -negative head and neck squamous cell carcinoma (HNSCC) tumors. (C) Relative median Treg infiltration across 
the 10 most immune-infiltrated tumors in increasing order including HPV-positive and -negative HNSCC tumors. (D) Relative median Treg/CD8+ T cell infiltration ratio across the 10 most immune-infiltrated tumors in increasing order including HPV-positive and -negative HNSCC tumors. (E) Relative median CD56 dim NK cell infiltration across the 10 most immune-infiltrated tumors in increasing order including HPV-positive and -negative HNSCC tumors. All box-and-whisker plots represent values within the interquartile range (IQR) (boxes) and $1.5 \times I Q R$ (whiskers). Outliers are plotted as values $>1.5 \times I Q R$ (circles).

shown to promote immune-mediated tumor clearance (38-41), effectors of innate immunity can lead to an inflammatory response and increase regional blood flow and angiogenesis, possibly contributing to tumor growth and progression (42).

One unanticipated exception to this overall trend was the favorable prognostic impact of Treg infiltration in HNSCC tumors: higher levels of Treg infiltration were associated with superior OS $(P=0.005$, adjusting for HPV status) (Figure 5C). The prognostic impact of Tregs in human cancer has been found to vary and depends not only on the individual tumor type but also on the subset of Tregs involved. In several other cancer types, Tregs have been associated with favorable prognosis (43). This positive prognostic aspect of Treg infiltration in certain contexts has been speculatively attributed to the ability of these cells to dampen inflammatory processes required for tumor survival/growth. However, these findings are in contrast with the vast majority of studies examining the impact of Tregs on prognosis, identifying poorer outcome in numerous studies across cancer types including melanoma, ovarian, breast, renal cell, lung, hepatocellular, and others (37). These latter findings are consistent with the immunosuppressive role of Tregs on effector T cells within the microenvironment.

We therefore hypothesized that in HNSCC, diverse T cell populations may be trafficked to the tumor microenvironment in concert with one another. Indeed, levels of tumor-associated Tregs correlated strongly with nearly all $\mathrm{T}$ cell populations, including $\mathrm{CD}^{+}\left(r=0.53, P=10^{-13}\right), \mathrm{CD}^{+} \mathrm{Th} 1(r$ $\left.=0.47, P=10^{-13}\right)$, T central memory $\left(r=0.37, P=10^{-10}\right)$, and T effector memory $\left(r=0.48, P=10^{-13}\right)$, but not Th2 cells $(r=0.08, P=0.14)$. We therefore examined the prognostic significance of Tregs in HNSCC, adjusting for the levels of immune cells that are suppressed by Treg activity, such as CD $8^{+}$ CTLs and NK cells, and controlling for HPV status, in multivariable analysis (Figure 5C). We found that, with adjustment for these other immune cells, Treg levels did not remain independently prognostic (HR $=0.21,95 \%$ CI 0.014-2.97, $P=0.25$ ) (Figure 5C and Supplemental Table 5).

These results indicate that Treg levels in HNSCC covary with broader trends among T cell populations, and that Treg levels in isolation may not represent the overall net level of immune activation or suppression in the tumor microenvironment. These findings agree with a model in which Tregs are shuttled to the tumor microenvironment (or differentiated to Tregs from conventional $\mathrm{CD}^{+} \mathrm{T}$ cells within the tumor microenvironment) in response to immune cytolytic activity (and associated cytokines, e.g., IL-2) in order to maintain immune homeostasis. In such a model, it is indeed the immune cytotoxicity mediated largely by $\mathrm{T}$ cells/NK cells that drives improved survival rather than the reactive presence of Tregs, which counter it. These findings have important clinical implications regarding the rationale of therapeutic strategies to target the Treg population in head and neck cancer (discussed further below).

Patients with tumors highly infiltrated with CD56 ${ }^{\text {dim }} \mathrm{NK}$ cells also experienced significantly improved survival as compared with patients with low CD56 dim infiltration (Figure $5 \mathrm{D})(P=0.007)$. In contrast, there was no prognostic significance associated with $\mathrm{CD} 6^{\text {bright }} \mathrm{NK}$ cell infiltration $(P=0.87)$. These data confirm the potential therapeutic advantages of activating NK cells in HNSCC - a cancer type defined by extraordinarily high levels of NK cell infiltration in our analysis.

We next examined the prognostic implications of the molecular smoking signature and immune infiltration. As described above, a stronger tobacco smoking mutational signature was significantly associated with both higher mutational load and lower levels of immune infiltration. We examined the prognostic value of the smoking signature in a multivariate model adjusting for other critical clinical and pathologic covariates: HPV status, TP53 mutation status, and pathologic TNM stage. The smoking signature outperformed these clinical and molecular covariates: an increasing level of smoking-associated mutagenesis was associated with poorer survival $(P=0.005)$ (Supplemental Table 6).

We then categorized tumors into 4 categories, according to levels of $\mathrm{T}$ cell infiltration (above/below median) and smoking signature contribution to mutational processes (above/below median). This metric was significantly associated with outcome, adjusting for the above clinical and molecular covariates $(P=0.016)$. Tumors with high $\mathrm{T}$ cell infiltration and low smoking signature had the best OS, and tumors with low $\mathrm{T}$ cell infiltration and high smoking signature the poorest OS (Supplemental Figure 7). 
A CD8+ T cell infiltration (high/low)

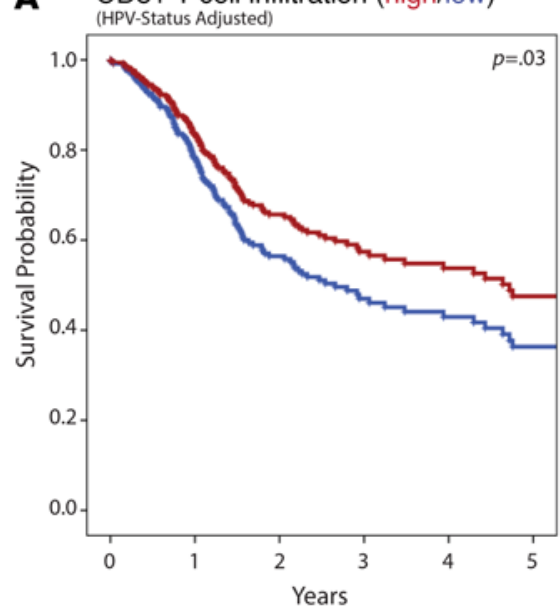

T cell infiltration (high/low)

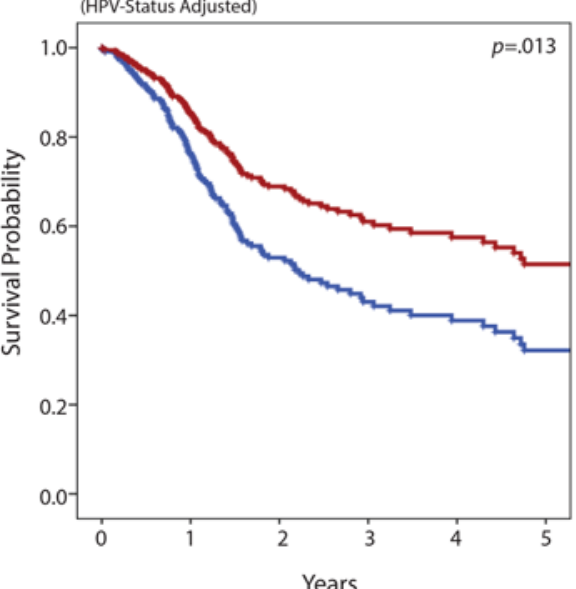

Overal immune infiltration (high/low)

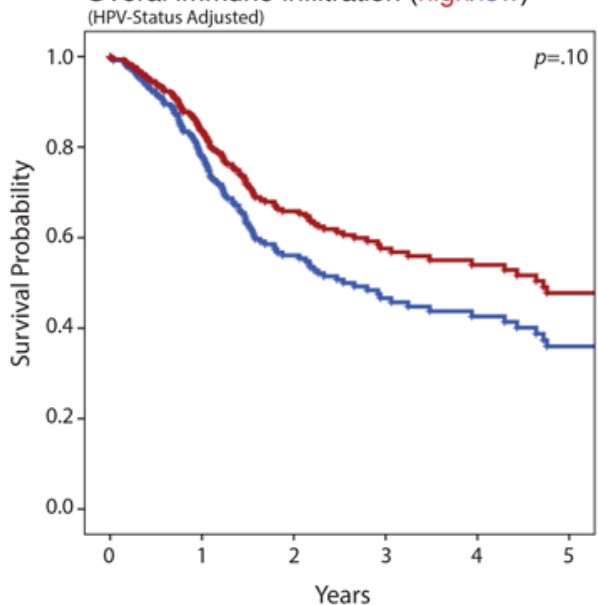

B

Immune subpopulations and survival

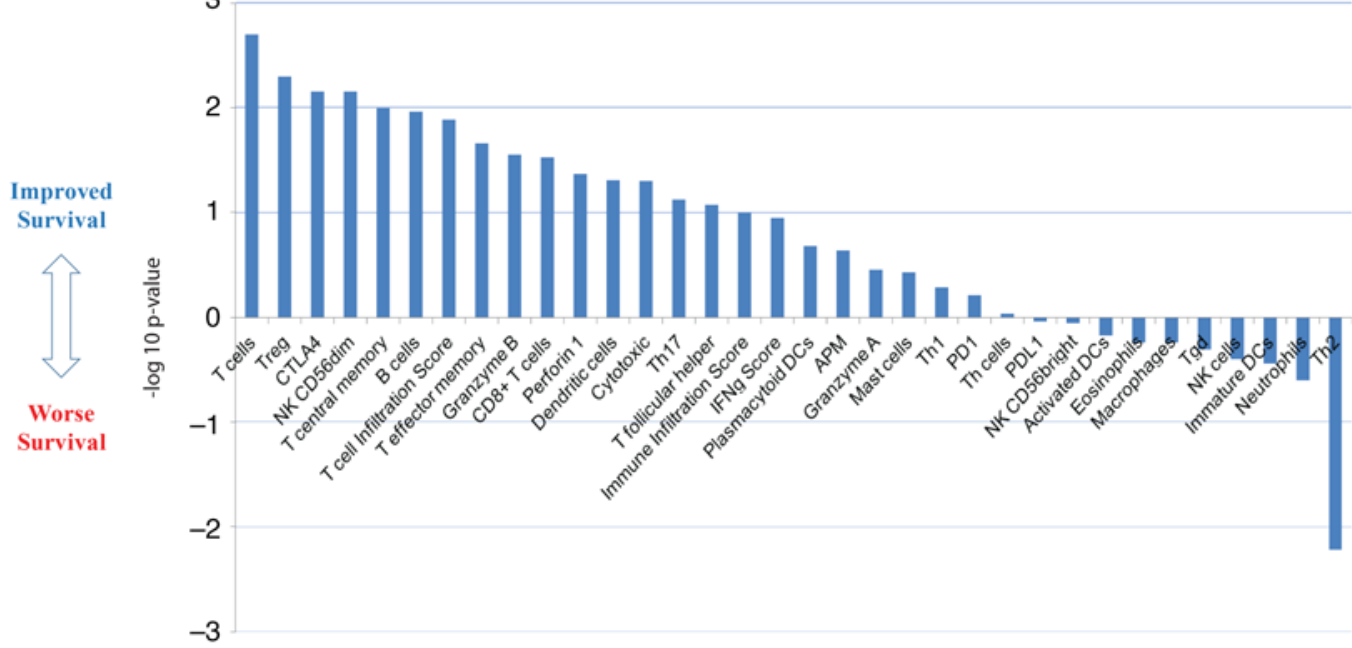

C

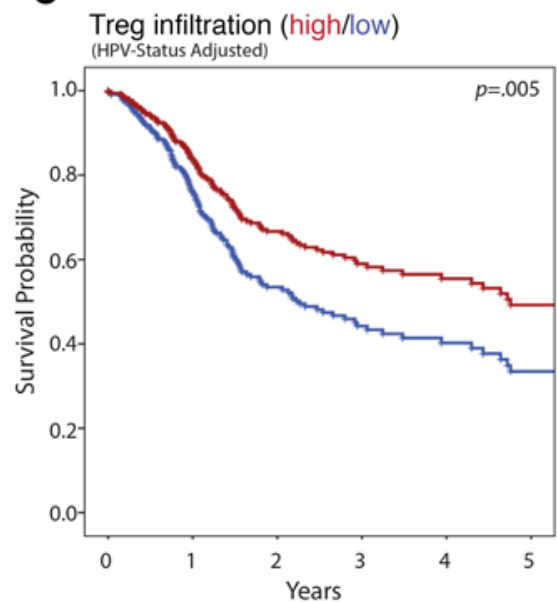

Treg (CTL, NK-cell independent) (high/low)

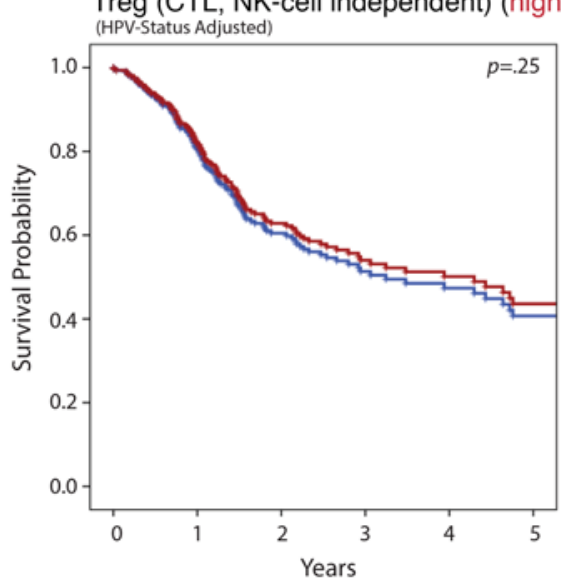

D

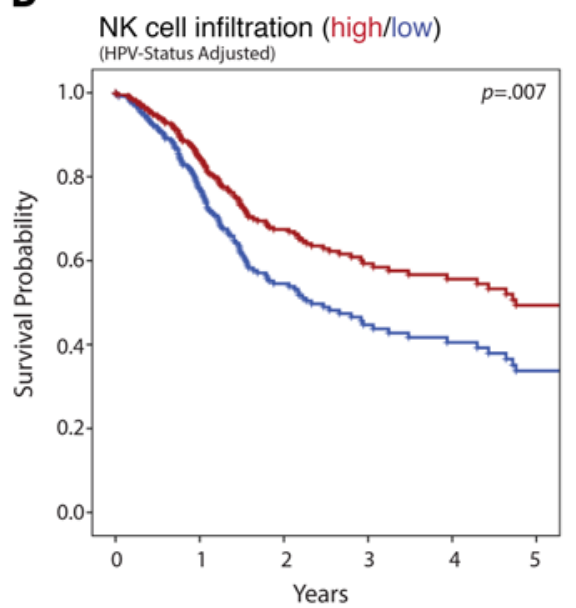

Figure 5. Head and neck squamous cell carcinoma immune infiltration and patient survival. (A) Correlations between expression levels plotted above or below the median of CD8 ${ }^{+} \mathrm{T}$ cell infiltration, overall T cell infiltration score (TIS), overall immune cell infiltration score (IIS), and patient cumulative survival. (B) Correlations between patient cumulative survival and immune cell subpopulations and immune effector agents represented by the -log10 $P$ value of the hazard ratio with more positive values indicating better survival and negative values indicating worse survival. (C) Correlation between expression levels plotted above or below the median of Treg infiltration and patient cumulative survival adjusted only for HPV status. Additional correlation between expression levels plotted above or below the median of Treg infiltration and patient cumulative survival adjusted for HPV status, CD8 $8^{+} \mathrm{TIS}$, and CD56 dim NK cell infiltration, abrogating the survival advantage for Tregs alone. (D) Correlation between high and low expression levels of CD56 dim NK cell infiltration and patient cumulative survival adjusted for HPV status. $P$ values for significance $(<0.05)$ calculated using multivariable Cox regression analysis. $n=280$. 


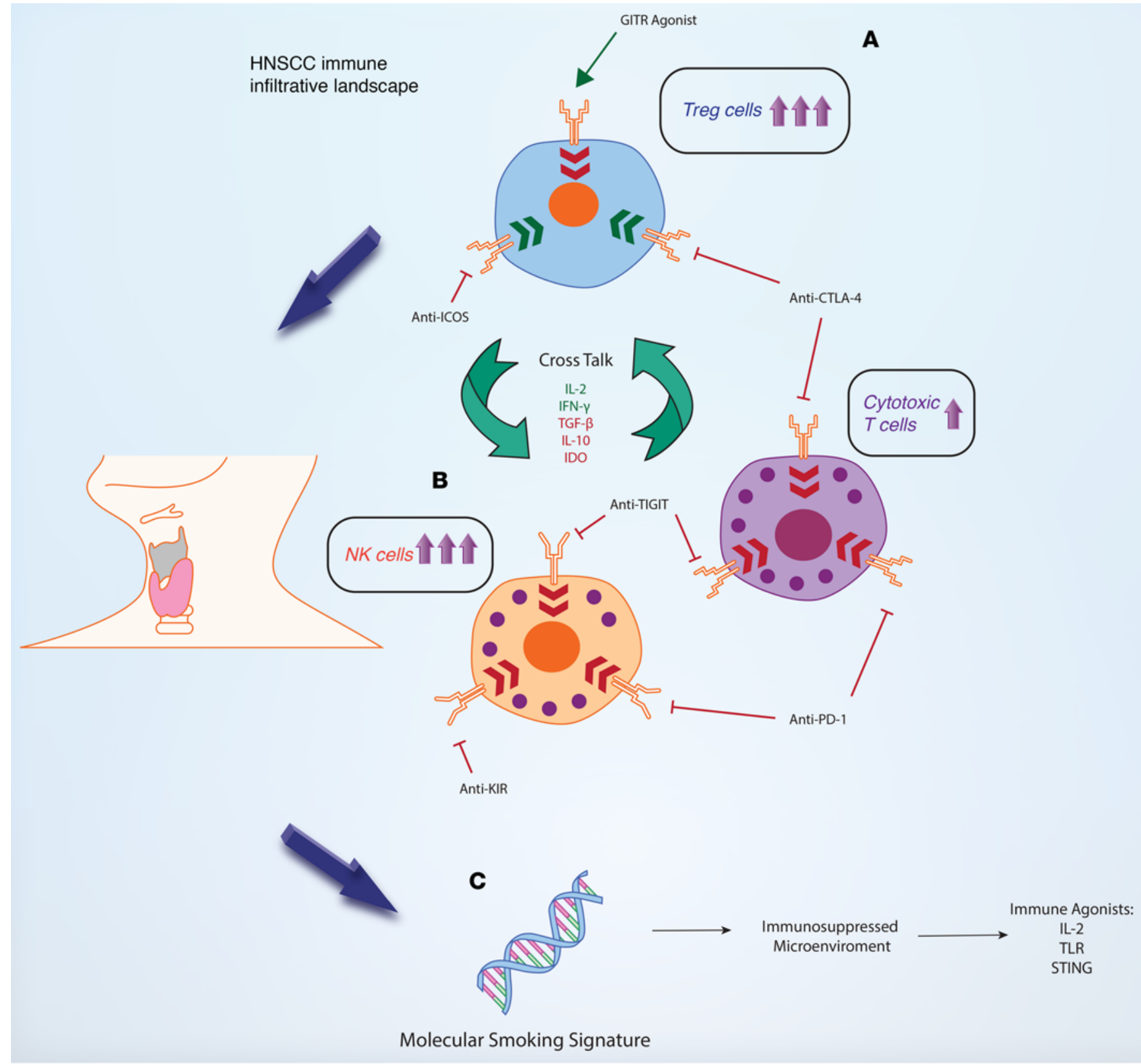

Figure 6. Head and neck squamous cell carcinoma (HNSCC) immune landscape and implications for immunotherapy. (A) HNSCC tumors possess the highest median Treg infiltration and the highest Treg/CD8 ${ }^{+} \mathrm{T}$ cell infiltration ratio of the 10 most immune-infiltrated tumors, providing a strong rationale to target modulators of Treg function such as CTLA-4, GITR, ICOS, IDO, VECFA, and others in head and neck cancer immunotherapy. (B) HNSCC tumors possess the highest median CD56 dim NK cell infiltration of the 10 most immune-infiltrated tumors providing, an additional rationale to augment NK cell activity in head and neck cancer via modulation of suppressive and agonistic receptors found on NK cells such as KIR, PD-1, TIGIT, 4-1BB, and others. (C) Patients displaying molecular smoking signatures possessed lower immune infiltration of their tumors and displayed worse survival, suggesting these patients may benefit from immune stimulatory therapy such as IL-2, TLR, and STING agonists. Green arrows indicate stimulatory signals and red arrows indicated inhibitory signals.

Immune infiltration and genomic instability. Measures of immune infiltration or activation did not correlate with tumor mutational load $(r=-0.034, P=0.58$ ) (Supplemental Table 7). However, there were strong inverse correlations between copy number alteration (the fraction of a tumor's genome that is copy-number altered, with a $\log _{2}$ copy number ratio $<-0.2$ or $>0.2$ ) and measures of immune infiltration, such as $\mathrm{T}$ cell infiltration, immune cell infiltration, and IFN- $\gamma$ signaling (Supplemental Figure 8), $r=-0.44,-0.46,-0.51$; all $\left.P<1 \times 10^{-4}\right)$. Senovilla et al. have demonstrated in immunocompetent mouse models that chromosomally unstable cells become immunogenic from endoplasmic reticular stress and subsequent increased calreticulin exposure (44). Consequently, these cells are subjected to immunoediting; specifically, hyperploid cells 
facilitate more efficient priming of $\mathrm{T}$ lymphocytes against tumor antigens and are subsequently eliminated by immune surveillance mechanisms (44). Such a process is consistent with the data observed in HNSCC, where more immune-infiltrated tumors have fewer copy number-altered cells. Conversely, highly copy number-altered tumors display low levels of immune infiltration, consistent with a lack of immune editing in these tumors. We repeated these analyses, stratifying by HPV status, and observed very similar results (Supplemental Table 8). To confirm that variations in tumor purity were not confounding this analysis, we performed multivariable analyses, controlling for tumor purity as determined by 2 independent methods (copy number-based ASCAT and expression-based ESTIMATE tools) $(45,46)$. In all cases, the inverse relationship between immune infiltrate and copy number alteration remained significant (Supplemental Table 9). Using the expression profiles in ESTIMATE, we confirmed that copy number alteration in tumors was inversely correlated with immune cell infiltration $(r=-0.15, P=0.009)$ but not with stromal infiltration $(r=$ $0.04, P=0.49$ ). We additionally used these tools to verify tumor purity by anatomical subsites to ensure that the measured immune infiltration was indeed resulting from the tumor and not surrounding tissue (Supplemental Figure 9).

These hypothesis-generating data require further mechanistic investigation. Although we have adjusted for variation in tumor purity, it is possible that there are other confounding covariates. Nevertheless, these data would be consistent with a process in which highly copy number-altered tumors represent aneuploid tumor cells that have escaped host immunity and have not undergone significant immunoediting. In line with such a hypothesis, we find that highly copy number-altered HNSCC tumors had poorer survival, and that C-class (copy number alteration-dominated) tumors were associated with poorer survival than M-class (mutation-dominated) tumors (Supplemental Figure 10).

We observe similar correlations when we examine a specific chromosomal marker of genomic instability in HNSCC. Chromosome $3 p$ deletion is a strong negative prognostic molecular feature in HNSCC (47). We found that deletion of $3 p$ was strongly associated with lower levels of $\mathrm{T}$ cell infiltration $(P=1 \times$ $\left.10^{-4}\right)$, immune cell infiltration $\left(P=2 \times 10^{-4}\right)$, IFN- $\gamma$ signaling $\left(P=5 \times 10^{-5}\right)$, and cytolytic score $(P=0.001)$, controlling for HPV status (Supplemental Table 10).

\section{Discussion}

Head and neck cancer currently represents one of the most promising areas of immunotherapy research. Despite approaches to date having been largely empirical, early data show a strong signal of activity for immune checkpoint blockade in these tumors. A rational approach to further clinical investigation requires a deeper understanding of the immune landscape of these cancers.

Here, we have analyzed immune infiltration in a large cohort of head and neck tumors, integrating genetic data with RNA-seq-based deconvolution of immune cell populations and effector/regulatory molecules. We find that HNSCC is one of the most highly immune-infiltrated cancer types, and in fact, the most highly NK cell- and Treg-infiltrated cancer type. We identified broad diversity in the levels of immune infiltration and activation across head and neck tumors, which varies based on clinical and genetic features, such as HPV status, tumor location, molecular subtype, mutational smoking signature, and genomic instability. These measures of immunity appear to represent clinically relevant processes, as they are highly correlated with patient outcome. We find that head and neck tumors have a distinct immune composition (differing from even similar tumor histological types such as lung squamous cancer) and this immune composition may dictate response to immunotherapies.

Several unique aspects of the immune infiltrative landscape of HNSCC are highly relevant to ongoing investigation in immuno-oncology (Figure 6): (a) Smoking-signature-high, HPV-negative HNSCCs have high mutational loads, but low levels of immune infiltration and activation. These tumors have poorer clinical outcome, after adjustment for HPV and other clinical and molecular factors. These immunologically cold but mutation/neoantigen-rich tumors may therefore benefit from immune agonists in combination with immune checkpoint blockade. (b) HPV-positive and HPV-negative HNSCC tumors are among the most highly immune-infiltrated cancer types, and possess the highest degrees of Treg infiltration. These tumors are poised to benefit from immunotherapy that includes Treg-targeted approaches. (c) Both HPV-positive and HPV-negative HNSCC have the highest levels of NK cell infiltration, suggesting that immunotherapeutic approaches inhibiting NK cell checkpoints may have unique applicability in head and neck cancer.

Ongoing trials with immune checkpoint inhibitors in HNSCC have shown promising preliminary results. A $17.7 \%$ overall response rate (ORR) has been observed in the ongoing KEYNOTE 012 
(NCT01848834) clinical trial of 192 patients with the recurrent/metastatic HNSCC treated with the antiPD-1 agent pembrolizumab (16). In this cohort, ORR in HPV-positive and -negative tumors was 21.9\% and 15.9\%, respectively (16). Most recently, the CheckMate-141 (NCT02105636) randomized phase III clinical trial demonstrated improved OS in patients with recurrent or metastatic platinum-refractory HNSCC treated with the anti-PD-1 agent nivolumab, compared with those treated with investigator's choice of either cetuximab, methotrexate, or docetaxel (17). OS in HPV-positive patients treated with nivolumab was 9.1 months versus 4.4 months in the control arm, whereas the OS in HPV-negative patients was 7.5 months versus 5.8 months, respectively (17). Interestingly, the improved response rates seen in HPV-positive tumors may be partially related to their higher immune infiltration, as we observe.

These results have led the FDA to grant a breakthrough therapy designation to nivolumab as a treatment for patients with recurrent or metastatic HNSCC. These studies indicate that durable clinical benefit can be observed in a substantial fraction of HNSCCs treated with immune checkpoint inhibitors, but that the majority of tumors nevertheless remain treatment resistant. In fact, the ORR in nivolumab-treated HNSCC patients was $13.3 \%$ with 1-year progression-free survival less than $10 \%$ (18). Deciphering the mechanistic basis of upfront treatment resistance, and tailoring the next steps of investigation, will require a more detailed understanding of the immune infiltrative landscape of these tumors.

There are 2 requirements for successful response of a tumor to immune checkpoint blockade. The first requirement is immune cell infiltration and recognition of tumor cells by the immune system. Immune cells must first overcome the interstitial pressures exerted by the tumor itself for successful infiltration $(48,49)$. Fortunately, head and neck tumors, in general, occur in richly lymphovascular sites (such as the oropharynx and cervical lymph nodes) and thus have the opportunity to interface with the full extent of the immune system. This is consistent with our finding that HNSCC is among the most highly immuneinfiltrated cancer types. However, this alone cannot ensure successful interaction between the immune system (particularly the adaptive immune system) and the tumor. This requires recognition of appropriate antigens by the effectors of adaptive immunity (e.g., CTLs).

The second component of a successful sustained antitumor immune response requires freedom from the immunoinhibitory influences from the tumor itself and immunosuppressive cells. Collectively, these influences permit immunosubversion (50). T cell exhaustion occurs via $\mathrm{T}$ cell-specific intrinsic mechanisms such as the PD-1/PD-L1 pathway as well as other immunoregulatory receptors including CTLA-4, TIM-3, LAG-3, and several others. (51-54). Additionally, T cell exhaustion can result from T cell extrinsic pathways mediated by Tregs, M2 macrophages, and myeloid-derived suppressor cells (MDSCs). These cells exert their immunoinhibitory influence primarily through secretion of immunosuppressive cytokines. MDSCs and their subsets, while likely an important component of extrinsic $\mathrm{T}$ cell exhaustion in some head and neck tumors, lack well-accepted gene expression signatures and therefore were not included in our analysis. However, as our understanding of their immunosuppressive phenotypes and gene expression profiles develops over time, further investigation of their role in head and neck cancer may prove beneficial.

Our findings demonstrate that HNSCC tumors are among the most highly immune-infiltrated cancer types, and that this immune infiltration is associated with cytolytic activity and activation of effector signaling pathways. These findings also provide insight into the second requisite component of successful immunemediated tumor clearance - relieving immunoinhibitory influences in tumors. We show that there are high levels of immunoregulatory influence in HNSCCs, particularly HPV-positive tumors, which harbor the highest levels of Treg infiltration and the highest Treg/CD8 ${ }^{+} \mathrm{T}$ cell ratio across all cancers, suggesting that these tumors are poised to respond to immunotherapeutic modalities that relieve inhibitory pathways.

Treg function and proliferation rely on a number of targetable pathways. CTLA- 4 is highly constitutively expressed on Tregs. Its activation is necessary for Treg function and proliferation. CTLA-4 inhibitors have clearly been shown to promote antitumor immunity in melanoma (2). Blockade of the CTLA-4 receptor on activated Tregs may therefore dampen the immunosuppressive function of these cells in head and neck tumors. A growing body of evidence suggests that CTLA-4 blockade does, in some contexts, repress Treg function and accumulation in the tumor microenvironment (55-57). The effect of anti-CTLA4 therapy on Tregs therefore deserves further investigation in HNSCC. Early results from other Treg-rich tumors such as NSCLC have shown impressive early results with the combined use of anti-PD-1 and antiCTLA-4 therapy, albeit with small numbers of patients (58). It will be interesting to see if anti-CTLA-4 (despite its worse toxicity profile) promotes antitumor immunity in HNSCC and whether this process is in part mediated by suppression of the Treg population. 
In addition, there are a number of emerging therapies with the potential to target Tregs that may exhibit significant activity in HNSCC. These include inhibitors of indoleamine 2,3-dioxygenase (IDO), which decrease the proliferation and activation of Tregs by increasing bioavailable tryptophan within the tumor microenvironment (59). IDO may also inhibit NK cell function as well. The IDO1 inhibitor epacadostat is in clinical investigation in multiple solid tumors in combination with anti-PD-1 agents. Agonists of glucocorticoid-induced tumor necrosis factor-related receptor (GITR) (highly expressed on Tregs) have been shown to induce tumor regression, in part mediated by the depletion of Tregs in the tumor microenvironment and downregulation of Foxp3 expression (60). Similarly, inducible T cell costimulator (ICOS) is highly expressed on Tregs and its activation is implicated in Treg expansion and function (61). Antagonists (e.g., MEDI570) of this receptor are in clinical investigation for other cancers and if found beneficial may be useful as well in HNSCC by suppressing the Treg compartment (62). Additional means of targeting Tregs include anti-VEGFA agents, which have been shown to decrease Tregs and improve survival in renal cell carcinoma $(37,63)$. These agents are currently used in clinical investigation in head and neck cancer and may be useful in combination with checkpoint blockade to augment anti-Treg responses.

Head and neck cancers had the highest degree of infiltration of the antitumor CD56 ${ }^{\mathrm{dim}}$ NK cell subpopulation in our pan-cancer analysis. Tumor infiltration with NK cells correlated significantly with patient survival. A substantial body of evidence in mice and humans demonstrates clear associations between NK cell activity and tumor immune surveillance $(32,64-67)$. We therefore hypothesize that augmenting NK cell activity may be highly advantageous in HNSCC. Augmenting NK cell responses may also be particularly important in targeting head and neck cancers with defects in their antigen-processing machinery (APM) and have thus escaped adaptive immunity. Despite their inability to recognize antigen, NK cells still possess selectivity in which cells they target. This is mediated largely by their NKG family of receptors and their respective ligands, which are preferentially expressed on infected cells and tumor cells $(68,69)$. Recent work has also shown that the activating receptor CD137 (4-1BB), on NK cells, enhances cytotoxicity in the presence of cetuximab via antibody-dependent cell-mediated cytotoxicity (ADCC) (70). This has led to an ongoing phase $\mathrm{Ib}$ trial combining cetuximab with the 4-1BB agonist, urelumab, in patients with advanced HNSCC.

NK cells additionally possess a complex network of inhibitory receptors, including KIR, T cell immunoreceptor with Ig and ITIM domains (TIGIT), T cell Ig mucin receptor 3 (TIM-3), and lymphocyte activation gene (LAG-3). These receptors have been clearly linked to inhibiting NK cell-mediated activity in antiviral and autoimmune immunology $(32,71)$. We hypothesize that blockade of these receptors may be particularly beneficial in HNSCC, a cancer type characterized by strikingly high levels of NK cell infiltration.

Recent evidence has shown that the PD-1 receptor (commonly found on T cells) can also be found on activated NK cells $(72,73)$. The combination of anti-PD-1 with the other inhibitory receptors mentioned above may synergistically facilitate enhanced NK cell cytolytic activity. It is quite possible that targeting only 1 inhibitory receptor will be insufficient to release the inhibition on NK cells in all tumors. Other checkpoint receptors may be dynamically upregulated in an adaptive fashion similar to $\mathrm{T}$ cell immune checkpoint adaptive resistance recently observed in lung adenocarcinoma (74). Therefore, targeting multiple receptors on the NK cell population may be particularly useful in head and neck cancer.

The simultaneous targeting of Tregs and activation of NK cells may also be particularly beneficial in head and neck cancer. Tregs attenuate NK cells by the secretion of immunosuppressive cytokines, which decrease their function and trafficking into the tumor microenvironment. In carcinogen-induced mouse tumor models, depletion of Tregs led to antitumor immunity that was largely mediated by the NK cell population (75). In another study it was shown in ex vivo assays that depletion of Tregs with CTLA-4 restored NK cell-mediated ADCC in the presence of cetuximab. These studies suggest that innate immunity plays an important role in tumor clearance once freed from the immunosuppressive influence of Tregs, which is particularly relevant in HNSCC given its unique immune landscape.

Finally, we identified a subset of HNSCCs associated with a strong genetic signature of smoking. These tumors tend to have low levels of immune infiltration, but high mutational loads. Our data therefore suggest that tobacco-associated HPV-negative HNSCC may have characteristics that are both favorable (higher mutational load) and unfavorable (low immune infiltration) with regard to the effectiveness of immune checkpoint blockade. Our findings suggest that smoking signature-high HPV-negative HNSCC may uniquely benefit from approaches to augment the immune response.

In lung cancer, our group previously reported that anti-PD-1 therapy achieved higher response rates in tumors with higher mutational loads and transversion-high smoking signatures (but this lung cancer study 
did not examine levels of tumor immune infiltration) (25). Our current study suggests that the high mutational load (and the resultant high neoantigen count) that results from tobacco carcinogenesis may mediate responses to immune checkpoint blockade, despite an overall immunologically cold tumor microenvironment. Given this relatively immunosuppressed microenvironment, these neoantigen-rich tumors may be poised to respond to therapies that augment the immune response. These agents can globally enhance responses of host adaptive and/or innate immunity. We therefore hypothesize that smoking signature-high HNSCC may benefit from immune modulators such as IL-2, TLR, and STING agonists used in other cancer types to boost overall host immunity. This may facilitate the response to checkpoint blockade, similar to results in other recent studies (76). It is possible that checkpoint blockade alone could potentially drive increased immune infiltration in smoking signature-high tumors in a chemokine-dependent fashion. This increased immune infiltration (along with increased neoantigen burden) could partly explain the higher response rates to anti-PD-1 therapy seen in smoking signature-high lung cancer (25). Similarly, as data become available, it will be interesting to correlate molecular smoking signatures with response to immune checkpoint blockade in head and neck cancers.

In conclusion, HNSCC is a cancer type that is highly immune infiltrated, on par with melanoma, renal cell carcinoma, and NSCLC, and an excellent candidate for the investigation of immunotherapeutic approaches. We propose that molecular characterization of HNSCC tumors should be an integral correlative aspect of ongoing investigation, and that uniquely relevant aspects of HNSCC include molecular tobacco signatures and Treg and NK cell infiltration.

\section{Methods}

Data sources. The normalized gene-level RSEM-normalized RNA-seq data for the TCGA HNSCC cohort (12), as well as 9 other TCGA data sets, were downloaded from the UC Santa Cruz cancer genomics browser (https://genome-cancer.ucsc.edu) (77). Additional data sets, including exome sequencing data in level 3-curated MAF files, clinical data, and Affymetrix SNP6 copy number data, were also downloaded from the Broad Institute Firehose pipeline on 6/3/2015.

Gene signatures and scoring for infiltration/activity levels with sSGSEA. To determine the degree of immune cell infiltration in tumors, we used a previously described and validated computational technique (19). In brief, this method applies expression-based gene signatures of immune cell populations (78) to individual tumor samples using ssGSEA (79). ssGSEA computes an overexpression score for a gene signature by comparing the ranks of the genes in the signature with the ranks of all other genes in the transcriptome. We characterized the degree of immune infiltration in HNSCC and 9 additional TCGA cancer cohorts by running ssGSEA with 24 expression-based immune gene signatures, comprising 509 genes in total.

The immune cell populations identified using this deconvolution approach included cells involved in innate immunity (mast cells, neutrophils, eosinophils, macrophages, plasmacytoid DCs, inactivated DCs, activated DCs, CD56 ${ }^{\text {dim }} \mathrm{NK}$ cells, and CD56 ${ }^{\text {bright }} \mathrm{NK}$ cells) and in adaptive immunity (B cells, T follicular helper, Th1, Th2, Th17, Treg, T $\gamma \delta$, T effector memory, $\mathrm{T}$ central memory, and $\mathrm{CD}^{+} \mathrm{T}$ cells). This algorithm has been orthogonally validated in samples studied with immunofluorescence staining, with high rates of concordance (19). To provide further confirmation of this algorithm we cross-validated immune populations quantified by this method with another validated deconvolution algorithm (CIBERSORT), which uses a different computational technique: a leukocyte gene-signature matrix (LM22) (80). We found that levels of immune subpopulations in HNSCC tumors measured with either algorithm were strongly and significantly correlated (Supplemental Figure 11).

We analyzed correlations between immune cell infiltration and the APM using a defined 7-gene APM signature that consisted of MHC class I genes (HLA-A/B/C, $\beta 2 \mathrm{M}$ ) and genes involved in antigen processing (TAP1, TAP2, and TAPBP).

The $\mathrm{T}$ cell infiltration score (TIS) was defined as the mean of the standardized values for $\mathrm{CD}^{+} \mathrm{T}, \mathrm{T}$ central and effector memory, Th1, Th2, Th17, and Treg cells. The overall immune infiltration score (IIS) for a sample was defined as the mean of the standardized values for macrophages, DC subsets (total, plasmacytoid, immature, activated), B cells, cytotoxic cells, eosinophils, mast cells, neutrophils, NK cell subsets (total, CD56 ${ }^{\text {bright }}, \mathrm{CD} 56^{\mathrm{dim}}$ ), and all $\mathrm{T}$ cell subsets excluding $\mathrm{T}$ gamma delta and $\mathrm{T}$ follicular helper cells (19).

IFN- $\gamma$ signaling was scored with ssGSEA using the REACTOME IFN- $\gamma$ geneset (http://www. broadinstitute.org/gsea/msigdb/cards/REACTOME_INTERFERON_GAMMA_SIGNALING). The IFN- $\gamma$ gene set had minimal redundancy with the immune population genes (3 of 63 overlap with 509 
immune genes). The cytolytic (CYT) score was obtained from the data set of Rooney et al. (20). Two techniques were used to define the molecular signature of tobacco carcinogenesis: the number of transversions, categorizing tumors as transversion-high or -low (25); and the proportion of mutations attributable to the tobacco signature (signature 4) in the Wellcome Trust Sanger Institute (WTSI) framework developed by Alexandrov $(23,81)$.

Clinical data definitions and statistical analyses. Head and neck cancer molecular subtypes were defined based on TCGA annotations for HPV status and expression-derived subtype (12). The survival outcome modeled was OS, where events are defined as death of any cause, and time in days. All Kaplan-Meier survival curves are plotted with the $x$ axis ranging from 0 to 5 years. Analyses of prognostic covariates were performed with adjustment for HPV status and other relevant clinicopathologic covariates, using Cox multivariable regression.

SNP6 array data were used to determine copy number alteration. For each individual cancer type, arrays were processed together, quantile normalized, and median polished using Affymetrix Power Tools. Allele-specific intensities were determined with the bird-seed algorithm, and then segmentation performed with allele-specific piecewise constant fitting (ASPCF) for the Log R ratio and B allele frequency tracks.

Two-sided Mann-Whitney and Fisher exact tests were performed for nonparametric categorical comparisons. Two-tailed $t$ tests and 1-way ANOVA were performed for continuous comparisons, because ssGSEA scores are approximately normally distributed. The unsupervised clustering for tumor samples, immune cell types, genes, and proteins was performed with hierarchical clustering with Pearson correlation. Scatterplot regression lines were generated with LOESS regression.

\section{Author contributions}

Project conception and design: RM, TC, LM. Conducting experiments and acquiring data: RM, YS, AD, LM. Analyzing and interpreting the data: RM, YS, AD, TC, LM. Drafting manuscript: RM, LM. Manuscript review and revision: RM, YS, AD, JH, MD, NR, KL, IG, AH, TC, LM. Graphical and technical support: RM, YS, AD, MD.

\section{Acknowledgments}

This work was supported by NIH grant P30 CA008748 (to Memorial Sloan Kettering Cancer Center). R.M. was supported by NIH grant T32 CA009685. L.G.T.M. was supported by the Damon Runyon Cancer Research Foundation, NIH grant K08 DE024774, and the Society of MSKCC. T.A.C. was supported by Pershing Square Sohn Cancer Research Alliance and STARR Cancer Consortium.

Address correspondence to: Luc Morris or Timothy Chan, 1275 York Avenue, New York, New York 10065, USA. Phone: 212.639.3049; E-mail: morris1@mskcc.org (L. Morris). Phone: 646.888.2765; E-mail: chant@mskcc.org (T. Chan).

1. Hamid O, et al. Safety and tumor responses with lambrolizumab (anti-PD-1) in melanoma. N Engl J Med. 2013;369(2):134-144.

2. Hodi FS, et al. Improved survival with ipilimumab in patients with metastatic melanoma. N Engl J Med. 2010;363(8):711-723.

3. Herbst RS, et al. Predictive correlates of response to the anti-PD-L1 antibody MPDL3280A in cancer patients. Nature. 2014;515(7528):563-567.

4. Tumeh PC, et al. PD-1 blockade induces responses by inhibiting adaptive immune resistance. Nature. 2014;515(7528):568-571

5. Tykodi SS. PD-1 as an emerging therapeutic target in renal cell carcinoma: current evidence. Onco Targets Ther. 2014;7:1349-1359.

6. Torre LA, Bray F, Siegel RL, Ferlay J, Lortet-Tieulent J, Jemal A. Global cancer statistics, 2012. CA Cancer J Clin. 2015;65(2):87-108.

7. Haddad RI, Shin DM. Recent advances in head and neck cancer. N Engl J Med. 2008;359(11):1143-1154.

8. Vokes EE, Weichselbaum RR, Lippman SM, Hong WK. Head and neck cancer. N Engl J Med. 1993;328(3):184-194.

9. Siegel R, Ward E, Brawley O, Jemal A. Cancer statistics, 2011: the impact of eliminating socioeconomic and racial disparities on premature cancer deaths. CA Cancer J Clin. 2011;61(4):212-236.

10. Hammerman PS, Hayes DN, Grandis JR. Therapeutic insights from genomic studies of head and neck squamous cell carcinomas. Cancer Discov. 2015;5(3):239-244.

11. Vermorken JB, et al. Open-label, uncontrolled, multicenter phase II study to evaluate the efficacy and toxicity of cetuximab as a single agent in patients with recurrent and/or metastatic squamous cell carcinoma of the head and neck who failed to respond to platinum-based therapy. J Clin Oncol. 2007;25(16):2171-2177.

12. Cancer Genome Atlas Network. Comprehensive genomic characterization of head and neck squamous cell carcinomas. Nature. 2015;517(7536):576-582. 
13. Seiwert TY, et al. Integrative and comparative genomic analysis of HPV-positive and HPV-negative head and neck squamous cell carcinomas. Clin Cancer Res. 2015;21(3):632-641.

14. Agrawal N, et al. Exome sequencing of head and neck squamous cell carcinoma reveals inactivating mutations in NOTCH1. Science. 2011;333(6046):1154-1157.

15. Stransky N, et al. The mutational landscape of head and neck squamous cell carcinoma. Science. 2011;333(6046):1157-1160.

16. Mehra R,et al. Efficacy safety of pembrolizumab in recurrent/metastatic head neck squamous cell carcinoma (R/M HNSCC): Pooled analyses after long-term follow-up in KEYNOTE-012 [abstract 6012]. J Clin Oncol. 2016;34.

17. Gillison ML, et al. Nivolumab (nivo) vs investigator's choice (IC) for recurrent or metastatic (R/M) head and neck squamous cell carcinoma (HNSCC): CheckMate-141. Paper presented at: Proceedings of the 107th Annual Meeting of the American Association for Cancer Research; April 19, 2016; New Orleans, LA.

18. Ferris RL, et al. Further evaluations of nivolumab (nivo) versus investigator's choice (IC) chemotherapy for recurrent or metastatic (R/M) squamous cell carcinoma of the head neck (SCCHN): CheckMate 141 [abstract 6009]. J Clin Oncol. $2016 ; 34$.

19. Senbabaoglu Y, et al. The landscape of T cell infiltration in human cancer its association with antigen presenting gene expression [published online ahead of print September 1, 2015]. bioRxiv. doi: http://dx.doi.org/10.1101/025908.

20. Rooney MS, Shukla SA, Wu CJ, Getz G, Hacohen N. Molecular and genetic properties of tumors associated with local immune cytolytic activity. Cell. 2015;160(1-2):48-61.

21. Chung $\mathrm{CH}$, et al. Molecular classification of head and neck squamous cell carcinomas using patterns of gene expression. Cancer Cell. 2004;5(5):489-500.

22. Walter V, et al. Molecular subtypes in head and neck cancer exhibit distinct patterns of chromosomal gain and loss of canonical cancer genes. PLoS One. 2013;8(2):e56823.

23. Alexandrov LB, et al. Signatures of mutational processes in human cancer. Nature. 2013;500(7463):415-421.

24. Stämpfli MR, Anderson GP. How cigarette smoke skews immune responses to promote infection, lung disease and cancer. Nat Rev Immunol. 2009;9(5):377-384.

25. Rizvi NA, et al. Cancer immunology. Mutational landscape determines sensitivity to PD-1 blockade in non-small cell lung cancer Science. 2015;348(6230):124-128.

26. Snyder A, et al. Genetic basis for clinical response to CTLA-4 blockade in melanoma. NEngl J Med. 2014;371(23):2189-2199.

27. Le DT, et al. PD-1 blockade in tumors with mismatch-repair deficiency. N Engl J Med. 2015;372(26):2509-2520.

28. Chow L, et al. Antitumor activity of the Anti-PD-1 antibody pembrolizumab in subgroups of patients with recurrent/metastatic head and neck squamous cell carcinoma (R/M HNSCC): Exploratory analyses from KEYNOTE-012. Eur J Cancer. 2015;51(3):S579. doi: 10.1016/S0959-8049(16)31604-5.

29. Seiwert TY, et al. Antitumor activity and safety of pembrolizumab in patients (pts) with advanced squamous cell carcinoma of the head and neck (SCCHN): Preliminary results from KEYNOTE-012 expansion cohort [abstract LBA6008]. J Clin Oncol. $2015 ; 33$.

30. CheckMate -141, a Pivotal Phase 3 Opdivo (nivolumab) Head Neck Cancer Trial, Stopped Early [press release]. Princeton, NJ: Business Wire; January 28, 2016.

31. Vivier E, et al. Innate or adaptive immunity? The example of natural killer cells. Science. 2011;331(6013):44-49.

32. Morvan MG, Lanier LL. NK cells and cancer: you can teach innate cells new tricks. Nat Rev Cancer. 2016;16(1):7-19.

33. Vey N, et al. A phase 1 trial of the anti-inhibitory KIR mAb IPH2101 for AML in complete remission. Blood. 2012;120(22):4317-4323.

34. Benson DM, et al. A phase I trial of the anti-KIR antibody IPH2101 and lenalidomide in patients with relapsed/refractory multiple myeloma. Clin Cancer Res. 2015;21(18):4055-4061.

35. Poli A, Michel T, Thérésine M, Andrès E, Hentges F, Zimmer J. CD56bright natural killer (NK) cells: an important NK cell subset. Immunology. 2009;126(4):458-465.

36. Ang KK, et al. Human papillomavirus and survival of patients with oropharyngeal cancer. N Engl J Med. 2010;363(1):24-35

37. Fridman WH, Pagès F, Sautès-Fridman C, Galon J. The immune contexture in human tumours: impact on clinical outcome. Nat Rev Cancer. 2012;12(4):298-306.

38. Koebel CM, et al. Adaptive immunity maintains occult cancer in an equilibrium state. Nature. 2007;450(7171):903-907.

39. Shankaran V, et al. IFNgamma and lymphocytes prevent primary tumour development and shape tumour immunogenicity. Nature. 2001;410(6832):1107-1111.

40. Galon J, et al. Type, density, and location of immune cells within human colorectal tumors predict clinical outcome. Science. 2006;313(5795):1960-1964.

41. Hanahan D, Weinberg RA. Hallmarks of cancer: the next generation. Cell. 2011;144(5):646-674.

42. Mantovani A, Allavena P, Sica A, Balkwill F. Cancer-related inflammation. Nature. 2008;454(7203):436-444.

43. Gentles AJ, et al. The prognostic landscape of genes and infiltrating immune cells across human cancers. Nat Med. 2015;21(8):938-945.

44. Senovilla L, et al. An immunosurveillance mechanism controls cancer cell ploidy. Science. 2012;337(6102):1678-1684.

45. Van Loo P, et al. Allele-specific copy number analysis of tumors. Proc Natl Acad Sci USA. 2010;107(39):16910-16915.

46. Yoshihara K, et al. Inferring tumour purity and stromal and immune cell admixture from expression data. Nat Commun. 2013;4:2612.

47. Gross AM, et al. Multi-tiered genomic analysis of head and neck cancer ties TP53 mutation to 3p loss. Nat Genet. 2014;46(9):939-943.

48. Hamzah J, et al. Vascular normalization in Rgs5-deficient tumours promotes immune destruction. Nature. 2008;453(7193):410-414.

49. Ariffin AB, Forde PF, Jahangeer S, Soden DM, Hinchion J. Releasing pressure in tumors: what do we know so far and where do we go from here? A review. Cancer Res. 2014;74(10):2655-2662.

50. Zitvogel L, Tesniere A, Kroemer G. Cancer despite immunosurveillance: immunoselection and immunosubversion. Nat Rev Immunol. 2006;6(10):715-727.

51. Chen L. Co-inhibitory molecules of the B7-CD28 family in the control of T-cell immunity. Nat Rev Immunol. 2004;4(5):336-347.

52. Zou W, Chen L. Inhibitory B7-family molecules in the tumour microenvironment. Nat Rev Immunol. 2008;8(6):467-477.

53. Dong H, et al. Tumor-associated B7-H1 promotes T-cell apoptosis: a potential mechanism of immune evasion. Nat Med. 2002;8(8):793-800. 
54. Butte MJ, Keir ME, Phamduy TB, Sharpe AH, Freeman GJ. Programmed death-1 ligand 1 interacts specifically with the B7-1 costimulatory molecule to inhibit T cell responses. Immunity. 2007;27(1):111-122.

55. Peggs KS, Quezada SA, Chambers CA, Korman AJ, Allison JP. Blockade of CTLA-4 on both effector and regulatory T cell compartments contributes to the antitumor activity of anti-CTLA-4 antibodies. J Exp Med. 2009;206(8):1717-1725.

56. Selby MJ, et al. Anti-CTLA-4 antibodies of IgG2a isotype enhance antitumor activity through reduction of intratumoral regulatory T cells. Cancer Immunol Res. 2013;1(1):32-42.

57. Simpson TR, et al. Fc-dependent depletion of tumor-infiltrating regulatory T cells co-defines the efficacy of anti-CTLA-4 therapy against melanoma. J Exp Med. 2013;210(9):1695-1710.

58. Patnaik A, et al. Phase 1 study of pembrolizumab (pembro; MK-3475) plus ipilimumab (IPI) as second-line therapy for advanced non-small cell lung cancer (NSCLC): KEYNOTE-021 cohort D [abstract 8011]. J Clin Oncol. 2015;33.

59. Munn DH. Indoleamine 2,3-dioxygenase, Tregs and cancer. Curr Med Chem. 2011;18(15):2240-2246.

60. Schaer DA, et al. GITR pathway activation abrogates tumor immune suppression through loss of regulatory $\mathrm{T}$ cell lineage stability. Cancer Immunol Res. 2013;1(5):320-331.

61. Redpath SA, et al. ICOS controls Foxp3(+) regulatory T-cell expansion, maintenance and IL-10 production during helminth infection. Eur J Immunol. 2013;43(3):705-715.

62. Mandal R, Chan TA. Personalized oncology meets immunology: the path toward precision immunotherapy. Cancer Discov. 2016;6(7):703-713.

63. Adotevi $\mathrm{O}$, et al. A decrease of regulatory $\mathrm{T}$ cells correlates with overall survival after sunitinib-based antiangiogenic therapy in metastatic renal cancer patients. J Immunother. 2010;33(9):991-998.

64. Imai K, Matsuyama S, Miyake S, Suga K, Nakachi K. Natural cytotoxic activity of peripheral-blood lymphocytes and cancer incidence: an 11-year follow-up study of a general population. Lancet. 2000;356(9244):1795-1799.

65. Schantz SP, Shillitoe EJ, Brown B, Campbell B. Natural killer cell activity and head and neck cancer: a clinical assessment. $J$ Natl Cancer Inst. 1986;77(4):869-875.

66. Pross HF, Lotzová E. Role of natural killer cells in cancer. Nat Immun. 1993;12(4-5):279-292.

67. Talmadge JE, Meyers KM, Prieur DJ, Starkey JR. Role of natural killer cells in tumor growth and metastasis: C57BL/6 normal and beige mice. J Natl Cancer Inst. 1980;65(5):929-935.

68. Raulet DH, Gasser S, Gowen BG, Deng W, Jung H. Regulation of ligands for the NKG2D activating receptor. Annu Rev Immunol. 2013;31:413-441.

69. Kruse PH, Matta J, Ugolini S, Vivier E. Natural cytotoxicity receptors and their ligands. Immunol Cell Biol. 2014;92(3):221-229.

70. Kohrt HE, et al. Targeting CD137 enhances the efficacy of cetuximab. J Clin Invest. 2014;124(6):2668-2682.

71. Martinet L, Smyth MJ. Balancing natural killer cell activation through paired receptors. Nat Rev Immunol. 2015;15(4):243-254

72. Norris S, Coleman A, Kuri-Cervantes L, Bower M, Nelson M, Goodier MR. PD-1 expression on natural killer cells and CD8(+) T cells during chronic HIV-1 infection. Viral Immunol. 2012;25(4):329-332.

73. Benson DM, et al. The PD-1/PD-L1 axis modulates the natural killer cell versus multiple myeloma effect: a therapeutic target for CT-011, a novel monoclonal anti-PD-1 antibody. Blood. 2010;116(13):2286-2294

74. Koyama S, et al. Adaptive resistance to therapeutic PD-1 blockade is associated with upregulation of alternative immune checkpoints. Nat Commun. 2016;7:10501.

75. Teng MW, Ngiow SF, von Scheidt B, McLaughlin N, Sparwasser T, Smyth MJ. Conditional regulatory T-cell depletion releases adaptive immunity preventing carcinogenesis and suppressing established tumor growth. Cancer Res. 2010;70(20):7800-7809.

76. Fu J, et al. STING agonist formulated cancer vaccines can cure established tumors resistant to PD-1 blockade. Sci Transl Med. 2015;7(283):283ra52.

77. Cline MS, et al. Exploring TCGA Pan-Cancer data at the UCSC Cancer Genomics Browser. Sci Rep. 2013;3:2652.

78. Bindea G, et al. Spatiotemporal dynamics of intratumoral immune cells reveal the immune landscape in human cancer. Immunity. 2013;39(4):782-795.

79. Barbie DA, et al. Systematic RNA interference reveals that oncogenic KRAS-driven cancers require TBK1. Nature. 2009;462(7269):108-112.

80. Newman AM, et al. Robust enumeration of cell subsets from tissue expression profiles. Nat Methods. 2015;12(5):453-457.

81. Alexandrov LB, Stratton MR. Mutational signatures: the patterns of somatic mutations hidden in cancer genomes. Curr Opin Genet Dev. 2014;24:52-60 